УДК 621.77:621.91

Публикуется в порядке обсуждения

А.Д. Учитель /д.т.н./,

Национальная металлургическая академия Украины, г. Днепр, Украина

Ю.А. Малиновский /к.т.н./,

Криворожский колледж Национального авиационного университета, г. Кривой Рог, Украина

А.Н. Панченко /к.т.н./,

Национальная металлургическая академия Украины, г. Днепр, Украина

Д.Н. Дворецкий,

Е.В. Омельченко,

Криворожский колледж Национального

С.Ю. Даценко

авиационного университета, г. Кривой Рог, Украина

\title{
ИНТЕНСИФИКАЦИЯ ПРОЦЕССОВ МЕХАНИЧЕСКОГО ВЗАИМОДЕЙСТВИЯ ИНСТРУМЕНТА С ЗАГОТОВКОЙ ПРИ ВЫПОЛНЕНИИ ВЫСОКОТОЧНЫХ И ЭНЕРГОЕМКИХ ТЕХНОЛОГИЧЕСКИХ ОПЕРАЦИЙ МЕТОДАМИ ДАВЛЕНИЯ И РЕЗАНИЯ
}

\author{
A. Uchytel /Dr. Sci. (Tech.)/, \\ Yu. Malinovskii /Cand. Sci. (Tech.)/, \\ H. Panchenko /Cand. Sci. (Tech.)/, \\ D. Dvoretskyi, \\ E. Omelchenko, \\ S. Datsenko
}

National Metallurgical Academy of Ukraine, Dnipro, Ukraine Kryvorizkyi College of the National Aviation University, Kryvyi Rih, Ukraine

National Metallurgical Academy of Ukraine, Dnepr, Ukraine Kryvorizkyi College of the National Aviation

University, Kryvyi Rih, Ukraine

\section{INTENSIFICATION OF PROCESSES OF MECHANICAL COOPERATION OF INSTRUMENT WITH PURVEYANCE ATIMPLEMENTATION OF HIGH-FIDELITY AND POWER-HUNGRY TECHNOLOGICAL OPERATIONS METHODS OFPRESSURE AND CUTTING}

Цель: исследовать динамику взаимодействия поверхностных слоев заготовки с инструментом при выполнении высокоточных и энергоемких технологических операций по обработке металлов давлением и механической обработки резанием, а также выполнить поиск методов интенсификаичи указанных рабочих прочессов, позволяющих повысить качество готовых изделий и понизить энергоемкость технологических прочессов.

Методика. Для исследований принята модель, в которой инструмент условно представлен в виде жёсткого штампа, а деталь представлена в виде податливой плоской полосы при различных условиях опирания наружных слоев обрабатываемых изделий.

В работе использована гипотеза о том, что нелинейное трение является источником самовозбуждаюшихся колебаний между инструментом и деталью, которые приводят к возникновению

(c) Учитель А.Д., Малиновский Ю.А., Панченко А.Н., Дворецкий Д.Н., Омельченко Е.В., Даценко С.Ю., 2019 
завышенных усилий резания и получению обработанных поверхностей, в ряде случаев, не достаточно высокого качества.

Построены математические модели для описания деформированных поверхностных слоёв детали под воздействием критических нагрузок.

Рекомендованы методы повышения эффективности обработки изделий, которые сводятся к применению наложения вибраций с определёнными амплитудами и частотами, причём, воздействие этих вибрачий сводится к выводу и решению уравнений, совпадающих по форме с уравнениями для самовозбуждающийся колебаний.

Научная новизна заключается в том, что для описания прочесса волочения на волочильных установках и процесса механической обработки изделий на металлорежущих станках применена одна и та же расчетная модель и использован один и тот же математический аппарат. Для интенсификации рассматриваемых технологических процессов рекомендовано использовать наложение вибрации с оптимальной настройкой по частоте и амплитуде.

Практическая ценность работы заключается в том, что указанный метод интенсификации рабочих прочессов при оптимальном выборе параметров наложенных вибраций может быть успешно применен при волочении холодной и горячей прокатке, штамповке, обработке фрезерованием, строганием, точением, дроблением измельчением и бурением горных пород и других технологических прочессов.

Ключевые слова: волочение, прокатка, накатка, штамповка, заготовка, деталь, фрезерование, строгание, характеристика трения, деформаџионная волна, частота, период, модуль упругости, потеря устойчивости, критическая сила.

DOI: $10.34185 / 0543-5749.2019-5-6-94-113$

Постановка проблемы. Многие технологические процессы по холодной обработке материалов давлением, такие как прокатка, накатка штамповка, волочение и другие могут быть уподоблены взаимодействию жёсткого штампа и заготовки. Наиболее типичной технологической операцией по взаимодействию штампа и заготовки является волочение, где рабочие поверхности волоки могут быть уподоблены жесткому штампу, взаимодействующему с основанием (плоским или криволинейным) из податливого материала (рис. 1).

Для предварительного рассмотрения вопросов формообразования будем полагать, что обрабатываемые грани заготовки имеют протяжённую прямолинейную или плоскую форму.

Обращаясь к усилиям, действующим на заготовку, учитывая, что заготовка будет находиться под воздействием усилия волочения $T$, которое вызовет распределенную нормальную нагрузку по форме волоки и распределенную касательную нагрузку от сил трения между волокой и заготовкой, причём равнодействующая проекции распределенной нагрузки на продольную ось будет численно равна суммарной силе трения между инструментом и заготовкой, а также будет равна на усилию волочения $T$. Упрощённо будем полагать, что распределение нормальных напряжений по поверхности волоки происходит по треугольному закону (рис. 1).
Связь между проекцией силы трения на горизонтальную ось и $T$ равнодействующей распределенных нормальных нагрузок $N$ будет определяться законом трения

$$
T=f \cdot N,
$$

где $T$ - равнодействующая проекций сил трения на горизонтальную ось (численно равная усилию волочения); $N$-равнодействующая реакций нормального давления на поверхности волоки (и заготовки при их взаимодействии); $f$ - установившееся значение коэффициента трения между заготовкой и волокой.

При таком нагружении обрабатываемого материала на его поверхности до входа в волоку появляется деформационная волна сжатия, имеющая вид периодической функции, а после выхода из волоки волна растяжения, которая не влечет за собой изменения геометрической формы заготовки. В зависимости от вида обрабатываемого материала и формы инструмента, деформационная волна сжатия может иметь вид деформированной полосы с «синусоидальными» гребнями или деформированной оболочки с гофрированной поверхностью.

Упрощённо будем считать, что контакт рабочей поверхности инструмента с гранью заготовки происходит по полосе, ширина которой определяется условиями данной задачи и шириной инструмента, а её толщина определяется величиной степени обжатия заготовки. 
Отметим, что при работе волочильной установки могут быть недостаточно обоснованно выбраны форма рабочей поверхности заготовки, скорость процесса волочения, а также недостаточная подача смазки на инструмент и заготовку. Эти недочеты в организации технологического процесса могут привести к нежелательному эффекту, так называемому подрезанию заготовки. Подрезание приводит к снятию «стружки» на поверхности заготовки. Этот процесс на- поминает обработку деталей и заготовок на металлорежущих станках. Такая аналогия позволяет рассматривать эти разные технологические процессы с одних позиций. Общим в этих процессах является то, что их можно свести к конкретной задаче теории упругости для анизотропной среды, которая обусловлена поверхностной обработкой наружных волокон, причём границей раздела двух сред являются внутренние волокна сжатых слоев.

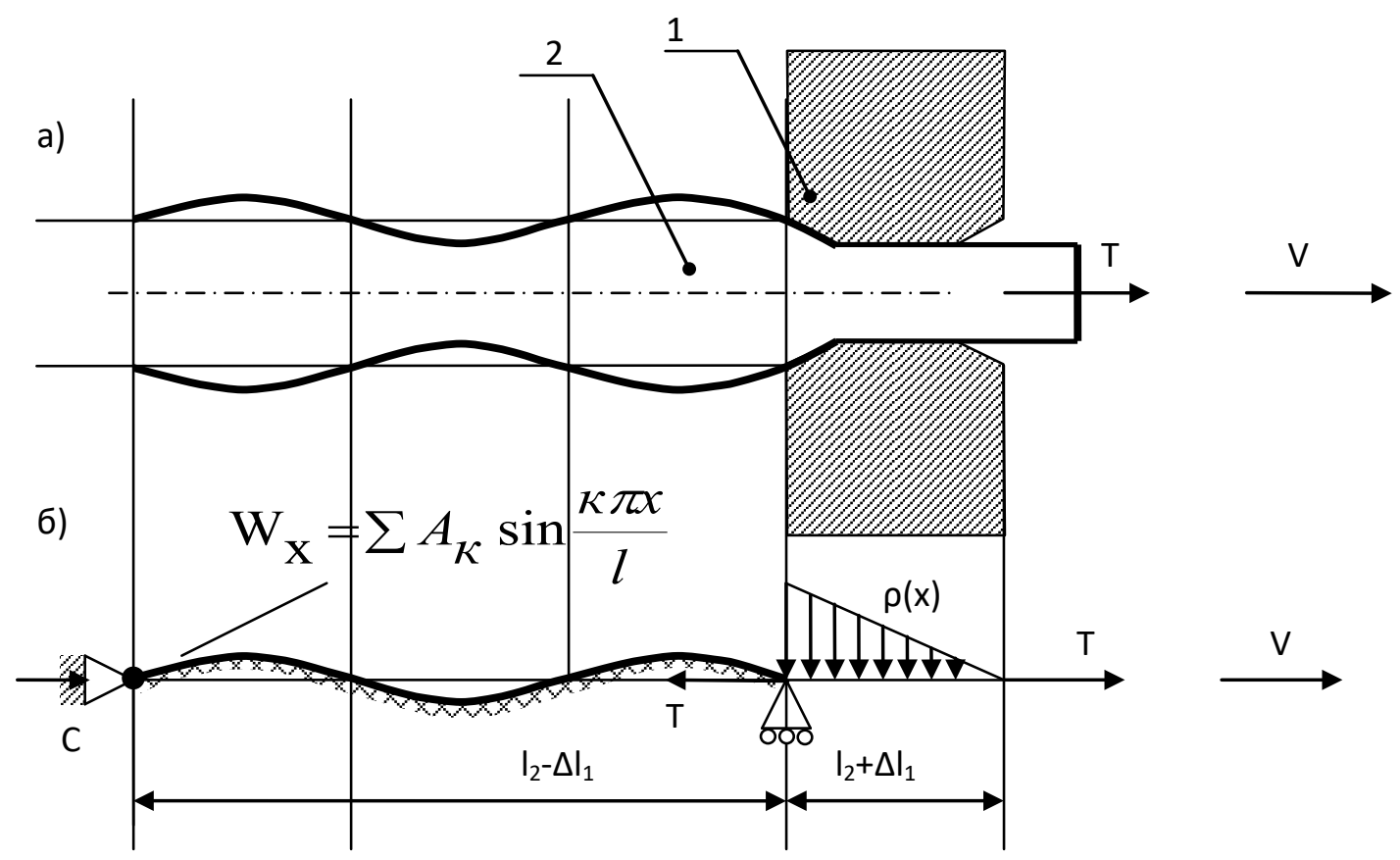

Рис. 1 Деформации поверхностных слоев заготовки при волочении: а) принципиальная схема волочения; б) расчетная схема поверхностных слоев. W - деформационная валка при волочении; $\mathrm{A}_{\kappa}$ - амплитуда деформационной гармоники с индексом - к; T - усилие процесса волочения; $1_{1}-$ длина деформационной части заготовки; $1_{2}$ - длина волоки; $\rho(\mathrm{x})$ - зависимость давления между волокой и заготовкой при волочении; 1 - волока; 2 - заготовка

В силу особенностей нагружения поверхности заготовки, эти слои логично представить в виде балки, толщина которой обусловлена степенью обжатия, ширина - шириной обрабатываемой грани (или может быть условно принята как балка единичной ширины). Такая условная балка обладает критической длиной, которая определяется длиной деформированного участка c расположенными на ней волнообразными микронеровностями.

При этом схема задачи принята в виде двухопорной балки на упругом основании. Заметим, что такая условная балка может быть разделена на зоны под штампом и перед штампом. В зоне под штампом (волокой) (рис. 1) происходит обжатие проталкиваемого материала, которое сопровождается интенсивным трением между взаимодействующими элементами.
Исходя из характера взаимодействия волоки и обрабатываемой заготовки (детали), возникающая деформационная волна сжатия верхних слоев обрабатываемого материала в зоне перед стационарной волокой (или роликовой волокой) при значительных степенях обжатия до $35-$ $42 \%$, формирует остаточные деформации, обусловленные местной потерей упругой устойчивости.

Отметим, что при пропускании заготовки через волоку, (например, неподвижную), происходит волнообразование по всем соприкасающимся граням инструмента и заготовки, сопровождающееся местной потерей устойчивости поверхностных слоев заготовки в зоне перед волокой. В этом случае, между инструментом и заготовкой имеют место эффекты, присутствующие при трении скольжения. Если же волочильная установка 
оборудована роликовой волокой, то в зоне перед инструментом произойдет волнообразование по всем взаимодействующим граням (например, четырём). В этом случае, между заготовкой и инструментом имеют место эффекты, присутствующие при трении качения.

Важной характеристикой операции волочения является величина коэффициента обжатия, которая ограничивается прочностью протянутой части и отсутствием самовозбуждающихся колебаний в зоне взаимодействия инструмента и заготовки. Из этих соображений следует, что для каждого пропуска заготовки необходимо определять усилие волочения. После определения усилия волочения необходимо проверить нагрузки на гранях волоки, которые могут привести к местной потере устойчивости наружных слоев заготовки и вызвать нежелательные параметрические или автоколебания этих слоев (которые часто являются причиной местных разрушений).

Все процессы, связанные с обработкой металлов давлением и, в ряде случаев, резанием, сопровождаются нестационарным трением в зоне взаимодействия инструмента и подаваемой заготовки [1].

Изложение основного материала. Нестационарной характер трения между инструментом и заготовкой зачастую приводит к раскачке неустойчивых колебаний в зоне их взаимодействия. Этот эффект наиболее выражен, если зависимость силы трения от скорости скольжения носит переменный характер с участками убывания и возрастания (точнее эта характеристика имеет «ниспадающую» ветвь). Обычно, такие самовозбуждающийся колебания называют автоколебаниями [1], [2]. На основании данных этих источников можно представить коэффициент трения скольжения $f=f(v)$ или силу трения $T=T(v)$ виде кубической зависимости от скорости скольжения заготовки по инструменту

$$
f=f_{0}-a v+b v^{3},
$$

где $f_{0}$ - значение коэффициента трения между инструментом и заготовкой в покое.

Из характеристики трения (рис. 2), найдём скорость скольжения, которой соответствует минимальное трение $f_{\min }$ при условии $\frac{d f}{d v}=0$ :

$$
f_{\text {min }}=f_{0}-a v_{\text {кр }}+3 b v_{\text {кр }}^{2},
$$

где $v_{\text {кр }}=\sqrt{\frac{a}{3 b}}-$ значение скорости скольжения (волочения), при котором достигается $f_{\min }$; $a, b-$ коэффициенты, характеристики трения с «ниспадающим» участком, которая строится по экспериментальным данным.

Коэффициенты $a$, $b$ удобно выразить в форме:

$$
\begin{aligned}
& a=3\left(f_{0}-f_{\text {min }}\right) / 2 v_{\text {кр }}, \\
& b=\frac{3}{2}\left(f_{0}-f_{\text {min }}\right) / 2 v_{\text {кр }}^{3} .
\end{aligned}
$$

Тогда, коэффициент трения можно представить в виде (для задач по обработке металлов давлением)

$$
f=f_{0}-\frac{3}{2}\left(f_{0}-f_{\min }\right) \frac{v}{v_{\mathrm{kp}}}+\left(\frac{f_{0}-f_{\min }}{2}\right)\left(\frac{v}{v_{\mathrm{kp}}}\right)^{3}
$$

Если каждое слагаемое (4) умножить на величину нормального давления $N$, то приходим к зависимости $T=T(v)$.

В приведенном выражении (3) $v_{k p}$ - значение скорости скольжения заготовки, при которой коэффициент трения $f$ (сила трения $T$ ) принимает значение $f_{\min }\left(T_{\min }\right)$.

При выполнении процесса волочения необходимо с одной стороны обеспечить минимальное трение между инструментом и заготовкой, и кроме того, как следует из работы [3], необходимо исключить автоколебания в системе, поэтому скорость протяжки $v_{\kappa p}$ обеспечит наименьшее трение между волокой и заготовкой, а если скорость протяжки будет превышать критическую $v_{n p} \geq 1,7 v_{\kappa p}$ тогда в системе автоколебания полностью отсутствуют.

Обращаясь к процессу взаимодействия инструмента и движущейся заготовки, нестационарная сила трения $T=T(v)$ одновременно действует на заготовку и инструмент вызывая при определённых условиях взаимодействия самовозбуждающиеся колебания (автоколебания) как инструмента и элементов его фиксации, так и автоколебания поверхностных слоев заготовки или отдельно автоколебания инструмента либо автоколебания поверхностных слоев заготовки.

Таким образом, в системе инструмент-движущаяся заготовка могут возникать автоколебания как инструмента, так и заготовки, однако в настоящей задаче важно избегать автоколебаний заготовки, так как они отрицательно влияют на качество обрабатываемой поверхности, способствуя раскачке параметрических колебаний наружных слоев заготовки.

Для исследования параметрических колебаний в наружных слоях заготовки опишем характеристику трения (рис. 2) в виде, предложенном Я.Г. Пановко [2]

$$
f=3 f_{\min }\left(1-\frac{v}{v_{\mathrm{\kappa p}}}+\frac{v^{3}}{3 v_{\mathrm{\kappa p}}^{3}}\right)
$$


или для сжимающих усилий

$$
T=3 T_{\min }\left(1-\frac{v}{v_{\text {кр }}}+\frac{v^{3}}{3 v_{\text {кр }}^{3}}\right) .
$$

При этом будем полагать, что переменная скорость скольжения при автоколебаниях определится

$$
v=v_{0}-\dot{x}
$$

где $v_{0}$ - номинальная скорость скольжения (точнее скорость волочения) заготовки; $\dot{x}$ - переменная скорость автоколебаний инструмента (волоки).

Тогда, выражение (6) с учётом выражения (7) запишем:

$$
T=T_{0}+T_{1} \dot{\mathrm{x}}+T_{2} \dot{\mathrm{x}}^{2}-T_{3} \dot{\mathrm{x}}^{3} .
$$

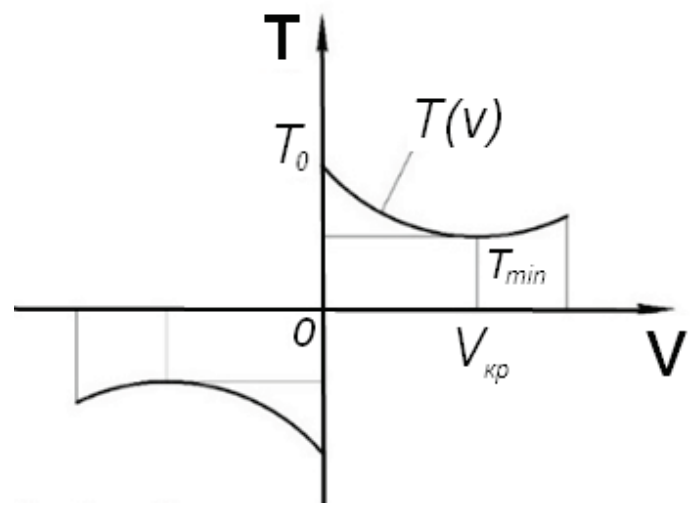

Рис. 2. Характеристика трения между инструментом и заготовкой: Т - сила (коэффициент) трения; $\mathrm{T}_{0}$ - сила трения при $\mathrm{V}=0$; $\mathrm{T}_{\min }-$ наименьшее значение силы трения при скорости скольжения (волочения) $\mathrm{V}=\mathrm{V}_{\text {кр }}$

В выражении (8) введены обозначения:

$$
\begin{gathered}
T_{0}=3 T_{\min }\left(1-\frac{v_{0}}{v_{\mathrm{\kappa p}}}+\frac{v_{0}^{3}}{3 v_{\mathrm{\kappa p}}^{3}}\right) ; \\
T_{1}=\frac{3 T_{\min }}{v_{\mathrm{\kappa p}}}\left(1-\frac{v_{0}^{2}}{v_{\mathrm{\kappa p}}^{2}}\right) ; \\
T_{2}=\frac{3 T_{\min } v_{0}}{v_{\mathrm{\kappa p}}^{3}} ; \\
T_{3}=\frac{T_{\min }}{v_{\mathrm{\kappa p}}^{3}} .
\end{gathered}
$$

Выражение (6) подставим в уравнение движения волоки (инструмента) при её автоколебаниях, которое имеет вид:

$$
m \ddot{x}+\left(\frac{d T}{d v}\right)_{0} \dot{x}+c x=T_{0},
$$

где $m$ - масса волоки с учетом массы элементов ее крепления; $c$ - приведенная жесткость волоки и элементов ее крепления; $T_{0}$ - номинальная сила трения между волокой и заготовкой (в состоянии покоя).
Если производная $\left(\frac{d T}{d v}\right)_{0}<0$, то в уравнении присутствует слагаемое, характеризующие отрицательное затухание, которое приводит к «раскачке» автоколебаний в системе «инструмент - заготовка».

Тогда, качественно, сила трения при автоколебаниях инструмента может быть описана выражением [2]:

$$
T=T_{0}+\left(\frac{d T}{d v}\right)_{0}\left(v-v_{0}\right)=T_{0}+\left(\frac{d T}{d v}\right)_{0} \dot{x} .
$$

Если решать уравнение (13) по методу гармонического баланса (или медленно меняющихся амплитуд) [2,] то приближённое решение (13) принимаем в виде:

$$
\begin{aligned}
& x=A \cos P_{k} t \\
& \dot{x}=-A P_{k} \sin P_{k} t .
\end{aligned}
$$

В приведенных выражениях соответственно $A, P_{k}$ - амплитуда и основная частота при автоколебаниях инструмента. Причём $A$ является медленно изменяющейся амплитудой.

Если переключиться на колебания наружных слоев заготовки, то они будут параметрическими, при этом, их раскачка будет происходить даже при $A=$ const, а закон изменения периодической силы в первом приближении будет иметь вид:

$$
T(t)=T_{0}+A P_{k} \sin P_{k}
$$

Выражение для $T(t)$ практически совпадает с выражением продольной силы для наружных слоев в виде:

$$
T(t)=T_{0}+T^{*} \sin P_{k}
$$

где $T^{*}$ - амплитудное значение переменной части сил трения, определяемое по (17); $P_{\kappa}-$ собственная частота системы «инструмент и его крепежные элементы», либо одна из собственных частот при продольных колебаниях поверхностных слоев

$$
P_{1} \sqrt{\frac{C}{m_{1}}} ; \quad P_{k+1}=\frac{(k+1)}{2 l} \sqrt{\frac{E F}{m_{2}}},
$$

где $m_{1}$ - приведенная масса инструмента с элементами его крепления; $c$ - жесткость упругой связи инструмента с элементами крепления; $E$ - модуль продольной упругости заготовки; $F$ - площадь поперечного сечения заготовки (рис. 3); $m_{2}$ - масса единицы длины заготовки; $l$ - длина участка заготовки, подверженного потере продольной устойчивости при волочении (или другой операции по механической обработке). 


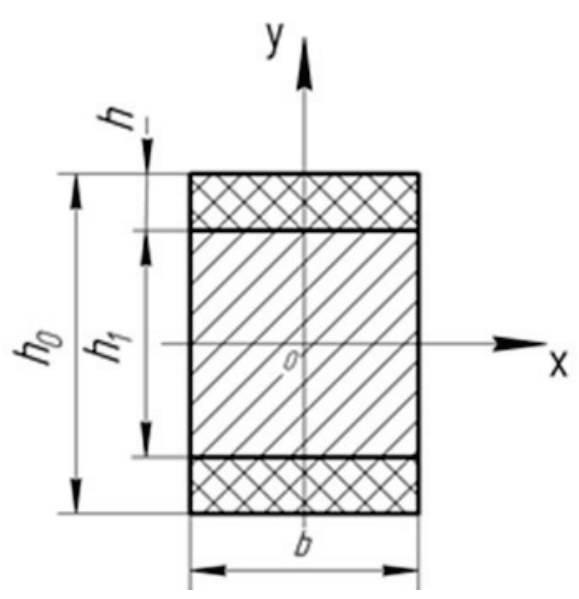

Рис. 3. К процессу волочения прямоугольной заготовки: $\mathrm{h}_{0}$ - полная высота заготовки; $\mathrm{h}$ - «толщина» деформируемого слоя; $\mathrm{h}_{1}$ - высота проходного сечения волоки; $\mathrm{b}-$ ширина заготовки

Выполним дальнейшие обозначения:

$r_{1}=\frac{T_{1}}{m_{1}} ; r_{2}=\frac{T_{2}}{m_{1}} ; r_{3}=\frac{T_{3}}{m_{1}} ; r_{0}=\frac{T_{0}}{m_{1}} ; P_{1}^{2}=\frac{c_{1}}{m_{1}}$,

тогда дифференциальное уравнение колебаний волоки (инструмента) преобразуем к виду:

$$
\ddot{x}-r_{1} \dot{x}-r_{2} \dot{x}^{2}+r_{3} \dot{x}^{3}+P^{2} x=r_{0} .
$$

Полученное дифференциальное уравнение является нелинейным, в котором присутствуют слагаемые, описывающие отрицательное затухание в системе. Если в (20) отбросить нелинейные слагаемые, то получим уравнение с отрицательным затуханием (слагаемое - $r, \dot{x}$ ). Решая такое линеаризованное уравнение, можно найти условия самовозбуждения колебаний (автоколебаний). Обращаясь к уравнению (20) в нелинейной постановке, согласно [2], можно воспользоваться приближенным решением уравнения по методу Ван дер Поля или методом энергетического баланса. По форме это решение совпадает с выражением (15). С учетом нелинейных слагаемых, решение уравнения запишем в виде:

$$
x(t)=\frac{A_{0} \cos P_{1} t}{\sqrt{\left(1-\frac{3 T_{3} P_{1}^{2}}{4 T_{1}} A_{0}^{2}\right) e^{-r_{1} t}+\frac{3 T_{2} P_{1}^{2}}{4 T_{1}} A_{0}^{2}} .}
$$

Тогда амплитуда колебаний инструмента $A_{1}$ может быть определена из выражения:

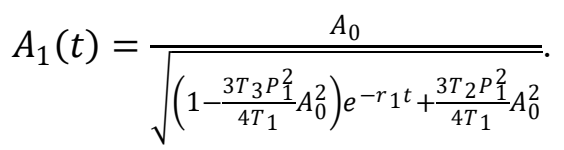

С течением времени все огибающие перио- дической функции (21) стремятся к одним и тем же горизонтальным прямым, соответствующим стационарному режиму колебаний с постоянной амплитудой

$$
A_{1}=\frac{2}{P} \sqrt{\frac{T_{1}}{T_{0}}}=2 \sqrt{\frac{m T_{1}}{c T_{0}}} .
$$

Также значение установившейся амплитуды можно представить в виде:

$$
A_{1}=2 v_{\min } \sqrt{\frac{m}{c} \frac{T_{1}}{T_{0}}} .
$$

Таким образом, с увеличением скорости (скольжения) $v_{0}$ равен 0 и её приближением к

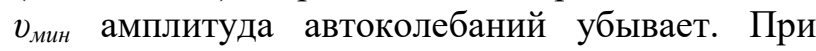
$v_{0} \geq v_{\text {мин }}$ автоколебания невозможны. С уменьшением скорости $v_{0}$ амплитуда колебаний растет и при некоторой достаточно малой скорости $v_{0}$ скорость скольжения $v=v-\dot{x}$ может обратиться в 0, то есть движение материала через волоку будет проходить с остановками, иначе будут иметь место разрывные колебания. Такой процесс механической обработки заготовки, с технологической точки зрения, будет неприемлем.

Отметим, что начальную амплитуду $A_{0}$ в формуле (22) можно трактовать как статическое смещение инструмента (волоки) $A_{0}=\frac{T_{0}}{c}$.

Таким образом, установлен вид продольной нагрузки для сжатых поверхностных слоев заготовки, которая представлена формулой (14). Перед рассмотрением деформирования поверхностных слоев заготовки исходим из того, что приняв шарнирное опирание гипотетической балки, как в волоке, так и на другом конце гипотетической балки (в месте затухания деформационных поверхностных волн), собственные функции задачи могут быть приняты в виде суперпозиции синусоидальных волн. При такой схематизации поверхностных слоев заготовки имеет место либо упругое оттеснение фрикционных связей как на макро- так и на микроуровнях при количестве циклов нагружения $n=10^{6} \div 10^{8}$, тогда после снятия нагрузки деталь полностью восстанавливается полностью восстанавливает свою форму. Либо пластическое оттеснение фрикционных связей (при числе циклов $n=1<10^{3}-10^{4}$ ). В этом случае, поверхностные слои детали теряют свою первоначальную форму и приобретают вид необратимой синусоидальной волны. Для этих двух видов фрикционных связей присутствует либо слабая смазка, либо сухое трение (рис. 4). 


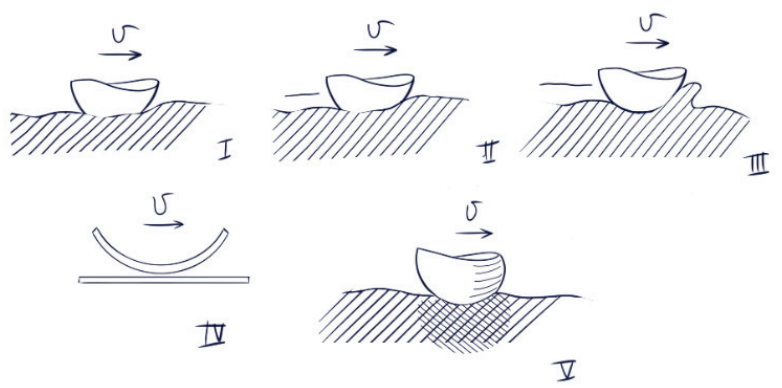

Рис. 4. Основные виды нарушения фрикционных связей при взаимодействии жесткой микронеровности с податливым основанием: I - упругое оттеснение; II - пластическое оттеснение; III - микрорезание; IV - адгезионный отрыв; V - когезионный отрыв

Тогда описание деформированных слоев заготовки в области перед волокой сведется к рассмотрению дифференциального уравнения [3]:

$$
\begin{gathered}
E_{n} I \frac{d^{4} W}{d x^{4}}+\left(T_{0}+T^{*} \sin P_{k} t\right) \frac{d^{2} W}{d x^{2}}+ \\
+\beta W+m \frac{d^{2} W}{d x^{2}}=0
\end{gathered}
$$

где $w(x, t)=W_{k}(t) \sin \frac{k \pi x}{l}$ - форма прогибов для поверхностных слоев заготовки при потере ими упругой устойчивости; во время волочения; $\sin \frac{k \pi x}{l}$ - собственные функции задачи, удовлетворяющие как уравнению, так и граничным условиям (при шарнирном опирании концов); $\max W_{\kappa}(t)=A_{\kappa}$ - амплитудное значение функции при $k=1,2 \ldots ; W_{\kappa}(t)$ - совокупность неизвестных функций времени, подлежащих определению; $T(t)$ - функция изменения продольной силы, причём $T_{0}$ - установившееся значение продольной силы, близкое к своему критическому значению $T_{0} \approx T_{\kappa p} ; \beta$ - жесткость упругого основания (или внутренних слоев материала); $I=\frac{b h}{12}$ момент инерции упрочненного слоя заготовки (рис. 3); $b$ - ширина упрочненного слоя заготовки (рис. 3 ); $h_{0}$ - полная высота гипотетической балки (заготовки); $h$-высота упрочненного слоя; $h_{1}$ - высота проходного сечения волоки (высота полосы после калибровки заготовки в волоке) (рис. 3); $l$ - длина деформированного участка заготовки в зоне перед волокой (величина неизвестная, подлежащая определению); $k$ - число полуволн на деформированном участке заготовки длиной $l$ (величина подлежит определению); $E_{n}$ - модуль упругости поверхностных слоев заготовки, в связи со сложным поверхностным эффектом в зоне взаимодействия инструмента и заготовки, является переменной величиной, так называемым приведенным модулем, который, согласно [4], принимается равным:

$$
E_{n}=\frac{E_{1} E_{\tau}}{\left(\sqrt{E_{1}}+\sqrt{E_{\tau}}\right)^{2}}
$$

где $E_{1}$ - модуль упругости равный начальному модулю; $E_{\tau}$ - касательный модуль равен наклону угла касательной; $\mathrm{T}^{*}$ - половина амплитуды колебаний нагрузки для одной из собственных частот.

Итак, если поверхностные слои заготовки испытывают действия возрастающей нагрузки, то эти слои имеют целую область критических значений [4]

$$
T_{\tau} \leq T_{\text {кр }} \leq T_{n} .
$$

То есть критическая сила для поверхностных слоев находится в диапазоне, пропорциональном изменению модуля упругости, что характерно для неустойчивых режимов работы и проявления параметрических эффектов в соответствии с уравнением (25).

Кроме того, при взаимодействии инструмента с заготовкой из-за внутреннего трения в материале заготовки происходит её саморазогрев в пределах тонких наружных слоев, который приводит к нарушению прямой пропорциональности между напряжением и деформацией при сжатии материалов $\sigma=\sigma(\varepsilon)=E \varepsilon^{\gamma}$, где $\gamma \neq 1$ - показатель степени.

Дифференциальное уравнение (25) рассматриваем с учётом граничных и начальных условий:

$$
\begin{aligned}
& w=\frac{d^{2} W}{d x^{2}}=0 \quad x=0 ; \\
& \frac{d W\left(x_{0}, 0\right)}{d t}=v_{0} x=l .
\end{aligned}
$$

После подстановки $w(x, t)$ в формулу (25) получим дифференциальные уравнения параметрических колебаний наружных слоев заготовки с переменными коэффициентами в форме Матье [5]:

$$
w_{k}^{\prime \prime}+\left[a_{k}+2 q_{k} \cos 2 \tau\right] w_{k}=0,
$$

где $a_{k}=\frac{4}{P_{k}^{2}}\left[\frac{E_{n} I}{m}\left(\frac{\pi k}{l}\right)^{4}-\frac{T_{0}}{m}\left(\frac{\pi k}{l}\right)^{2}+\frac{\beta}{m}\right]$; $2 q_{k}=\frac{4}{P_{k}^{2}}+\frac{T^{*}}{m}\left(\frac{\pi k}{2}\right)^{2}$.

При этом $t=2 \pi-\frac{\pi}{2}, \tau$ - новая временная переменная.

Обращаясь к дифференциальному уравнению (30), при условии, что усилие $T_{\approx} \approx T_{\kappa p}$ будет близко к критическому и в системе инструментзаготовка возникнут фрикционные автоколебания, согласно закону $x(t)=A_{0} \cos P t$, то в поверхностных слоях заготовки возникнет параметрический резонанс, процесс обработки становится 
неустойчивым и при этом, как минимум, форма обрабатываемой поверхности окажется низкого качества из-за изменений шероховатости и появления непрогнозируемых микротрещин.

Поэтому, при определении условий возникновения опасных параметрических колебаний предварительно необходимо вычислить значение критической сжимающей силы $T_{\kappa p}$, которая будет параметрическим критерием для оценки неустойчивых состояний поверхностных слоев заготовки.

Если продольная сила $T \geq T_{\kappa p}$, то форма поверхности заготовки при работе в упругой части характеристики материалаб $=\sigma(\varepsilon)$ приобретет вид гармонической функции, количество полуволн которой и их длина является неизвестными величинами $(k, l)$, тогда в исходное уравнение (25) подставим выражение $w(x, t)=W_{k} \sin \frac{k \pi x}{l}$, считая $t=$ const.

$$
\begin{gathered}
E_{n} I \frac{k^{4} \pi^{4}}{l^{4}} w_{k} \sin \frac{k \pi x}{l}-T_{\mathrm{кр}} \frac{k^{2} \pi^{2}}{l^{2}} \sin \frac{k \pi x}{l}+ \\
+\beta W_{k} \sin \frac{k \pi x}{l}=0,
\end{gathered}
$$

откуда

$$
\begin{aligned}
T_{\text {кр }} & =E_{n} I \frac{k^{4} \pi^{4}}{l^{4}}+\frac{\beta l^{2}}{k^{2} \pi^{2}}= \\
& =E_{n} I \frac{k^{2} \pi^{2}}{l^{2}}+\frac{E}{2} \frac{k \pi}{l} \frac{l^{2}}{l^{2} \pi^{2}} .
\end{aligned}
$$

При этом $\beta=\frac{E \alpha}{2}-$ жесткость упругого основания [6].

Таким образом $T_{\kappa p}$ является дискретной функцией числа полуволн $k$ на длине участка заготовки $l$. Нас интересует случай при каком значении $k$ получим минимальное значение критической силы $T_{k p}$. Условно примем, что в формуле (32) фигурирует непрерывная переменная $\alpha=\frac{k \pi}{l}$, которая представляет собой неизвестное число полуволн на участке заготовки длиной $l$. Тогда вместо (32) будем иметь:

$$
T=E_{n} I \alpha^{2}+\frac{\beta}{\alpha^{2}} \text {. }
$$

Далее находим минимум выражения $\frac{d T}{d \alpha}=0$. Получим:

$$
\begin{aligned}
& T=E_{n} I \alpha^{2}+\frac{E}{2 \alpha} ; \\
& \frac{d T}{d \alpha}=E_{n} I \alpha-\frac{E}{2} \alpha^{-2},
\end{aligned}
$$

откуда:

$$
\alpha=\sqrt[3]{\frac{E}{4 E_{A} I}} .
$$

В приведённых выражениях (34) и (36) $E$ модуль упругости внутренних (неуплотненных слоев заготовки).

Тогда из выражения $\alpha=\frac{k \pi}{l}$ следует:

$$
k=\frac{l}{\pi} \sqrt[3]{\frac{E}{4 E_{n} I}} .
$$

При этом длина участка заготовки, потерявшего упругую устойчивость пока не известна. Для её определения обратимся к выражению, связующему отношение критической силы для наружных слоев заготовки к их изгибной жесткости с числом полуволн $k$ деформированном участке согласно [7]:

$$
\begin{aligned}
\frac{T_{\mathrm{kp}}}{E_{n} I} & =\frac{\pi^{2}}{l^{2}}\left[k^{2}+(k+1)^{2}\right]= \\
& =\frac{\pi^{2}}{l^{2}}\left(2 k^{2}+2 k+1\right) .
\end{aligned}
$$

Учитывая значение $T_{\kappa p}$ в форме (32) будем иметь:

$$
\begin{gathered}
E_{n} I \frac{k^{2} \pi^{2}}{l^{2}}+\frac{E}{2} \frac{l}{k \pi}= \\
=\frac{\pi^{2}}{l^{2}} 2 k^{2} E_{n} I+\frac{\pi^{2}}{l^{2}} 2 k E_{n} I+\frac{\pi^{2}}{l^{2}} E_{n} I \\
\frac{E}{2} \frac{l}{k \pi}=\frac{\pi^{2}}{l^{2}} 2 k^{2} E_{n} I+\frac{\pi^{2}}{l^{2}} 2 k E_{n} I+\frac{\pi^{2}}{l^{2}} E_{n} I \\
l^{3}=k^{3} \pi^{3} \frac{2 E_{n} I}{E}+k^{2} \pi^{3} \frac{4 E_{n} I}{E}+A^{3} \frac{2 E_{n} I}{E} k \\
l^{3}=\frac{l^{3}}{\pi^{3}}\left(\sqrt[3]{\frac{E}{4 E_{n} I}}\right)^{3} \frac{\pi^{3} 2 E_{n} I}{E}+ \\
+2 \frac{l^{2}}{\pi^{2}}\left(\sqrt[3]{\frac{E}{4 E_{n} I}}\right)^{2} \frac{4 E_{n} I}{E}+\pi^{3} \frac{2 E_{n} I}{E} \frac{l}{\pi} \sqrt[3]{\frac{E}{E_{n} I}} \\
l^{2}=4 l \pi^{\frac{4 E_{n} I}{E}}+\pi^{2}\left(\sqrt[3]{\frac{4 E_{n} I}{E}}\right)^{2}=0
\end{gathered}
$$

откуда

$$
\begin{aligned}
& l_{1,2}=2 \pi \sqrt[3]{\frac{4 E_{n} I}{E}} \pm \sqrt{4 \pi^{2}\left(\sqrt[3]{\frac{4 E_{n} I}{E}}\right)^{2}-\pi^{2}\left(\sqrt[3]{\frac{4 E_{n} I}{E}}\right)^{2}} \\
& l_{1,2}=2 \pi \sqrt[3]{\frac{4 E_{n} I}{E}} \pm \sqrt{3} \pi \sqrt[3]{\frac{4 E_{n} I}{E}}= \\
& =\pi(2 \pm \sqrt{3}) \sqrt[3]{\frac{4 E_{n} I}{E}} .
\end{aligned}
$$

Согласно выражению (43) возможны два значения длины гипотетической балки (заготовки), потерявшей продольную устойчивость. Исходя из физического смысла, длина гипотетической балки, потерявшей упругую устойчивость, должна быть больше длины одной полуволны такой балки: 


$$
l_{1}=\pi(2 \pm \sqrt{3}) \sqrt[3]{\frac{4 E_{n} I}{E}}>\lambda=\pi \sqrt[3]{\frac{4 E_{n} I}{E}} .
$$

Тогда число полуволн деформированной части заготовки при однократном приложении критической силы $T_{\kappa p}$ для системы координат, движущейся со скоростью заготовки, должно быть равно:

$$
k=\frac{l}{\pi} \sqrt[3]{\frac{E}{4 E_{n} I}}=\frac{(2+\sqrt{3})}{\pi} \pi \sqrt[3]{\frac{4 E_{n} I E}{E 4 E_{n} I}}=(2+\sqrt{3})
$$

Выразим длину деформируемой части балки через физические и геометрические её параметры. При этом, будем исходить из того, что глубина накатанного слоя определяется разностью поперечных размеров исходной заготовки и поперечных размеров волоки. Обозначим глубину накатанного слоя $h_{1}$, тогда $I=\frac{b h_{1}^{3}}{12}$. В свою очередь, можно принять, что толщина накатанного слоя определяется согласно [8] по формуле:

$$
h_{1}=\sqrt{\frac{N}{2 \sigma_{T}}} .
$$

где $N$ - вертикальная нагрузка на волоку возникающая как реакция при трении заготовки через инструмент; $\sigma_{\text {т }}$ - предел текучести материала балки.

Подставим зависимость (46) в выражение (44), тогда будем иметь:

$$
\begin{gathered}
l_{1}=(2 \pm \sqrt{3}) \pi \sqrt[3]{\frac{4 E_{c} b}{12 E}\left(\sqrt{\frac{P}{2 \sigma_{T}}}\right)^{3}=} \\
=(2 \pm \sqrt{3}) \pi \sqrt{\frac{P}{2 \sigma_{T}}} \sqrt[3]{\frac{E_{c} I}{3 E}}
\end{gathered}
$$

Таким образом, получим формулу для определения критической длины условной балки состоящей из уплотненных слоев заготовки, которая зависит от вертикальной нагрузки, модулей упругости наружных и внутренних слоев, предела текучести материала и ширины балки. Критическая длина является такой длиной деформированной части заготовки, на которой могут происходить авто- и параметрические колебания, если скорость протяжки соответствует скорости для минимума коэффициента трения на ниспадающем участке характеристики (рис. 1).

Кроме того, параметрическая касательная нагрузка на заготовку (балку), то есть постоянная нагрузка при которой возможно возникновение неустойчивых колебательных процессов во внешних слоях балки, может быть определена как функция от указанных величин $P, \sigma_{T,} E_{c}$, $E, b$. С этой целью обратимся к формуле (34), подставляя в неё найденные выражения для $k, l$, $I$, и переходим к следующим зависимостям:

$$
\begin{aligned}
& T_{\text {кр }}=E_{C} I \frac{k^{2} \pi^{2}}{l^{2}}+\frac{E}{2} \frac{l}{k \pi}= \\
& =E_{c} I \frac{\pi^{2}}{l^{2}} \frac{l^{2}}{\pi^{2}}\left(\sqrt[3]{\frac{E}{4 E_{c} I}}\right)^{2}+\frac{E}{2} \frac{l}{k T}= \\
& =E_{c} I\left(\sqrt[3]{\frac{E}{4 E_{c} I}}\right)^{2}+\frac{E}{2} \frac{l}{\pi} \frac{\pi}{l} \sqrt[3]{\frac{4 E_{c} I}{E}} . \\
& T_{\text {кр }}=E_{C} I\left(\sqrt[3]{\frac{E}{4 E_{c} I}}\right)^{2}+\frac{E}{2} \sqrt[3]{\frac{4 E_{c} I}{E}}= \\
& =\sqrt[3]{\frac{E^{2}\left(E_{c} I\right)^{3}}{4^{2}\left(E_{c} I\right)^{2}}}+\frac{1}{2} \sqrt[3]{E_{c} I E^{2}}= \\
& =\frac{1}{2} \sqrt[3]{\frac{E^{2} E_{c} I}{2}}+\frac{1}{2} \sqrt[3]{\frac{8 E^{2} E_{c} I}{2}}= \\
& =\frac{1}{2} \sqrt[3]{\frac{E^{2} E_{c} I}{2}}+\frac{2}{2} \sqrt[3]{\frac{E^{2} E_{c} I}{2}}= \\
& =\frac{3}{2} \sqrt[3]{\frac{E^{2} E_{c} I}{2}}=\frac{3}{2} \sqrt[3]{\frac{E^{2} E_{c} b\left(\sqrt{\frac{P}{2 \sigma_{T}}}\right)^{3}}{2}}= \\
& =\frac{3}{2} \sqrt{\frac{N}{2 \sigma_{T}}} \sqrt[3]{\frac{E^{2} E_{c} b}{2}} .
\end{aligned}
$$

Окончательно:

$$
T_{\text {кр }}=\frac{3}{2} \sqrt{\frac{A N}{2 \sigma_{T}}} \sqrt[3]{\frac{E^{2} E_{c} b}{2}} .
$$

Определим критический коэффициент трения при достижении которого имеют место параметрические эффекты при волочении:

$$
\begin{aligned}
f_{\text {кр }}= & \frac{T_{\text {кр }}}{N}=\frac{3}{2} \sqrt{\frac{N}{2 N^{2} \sigma_{T}}} \sqrt[3]{\frac{E^{2} E_{A} b}{2}}= \\
= & \frac{3}{2} \sqrt{\frac{1}{2 N \sigma_{T}}} \sqrt[3]{\frac{E^{2} E_{A} b}{2}} .
\end{aligned}
$$

При таком значении коэффициента трения процесс волочения будет неустойчивым, возможно сначала проявление фрикционных автоколебаний, а затем и параметрических колебаний поверхностных слоев заготовки.

Таким образом, мы получили значение критической силы $T_{\text {кр}}$, критической длины участка заготовки $l$, потерявшей продольную устойчивость в зоне перед волокой, количество полуволн деформаций $k$ в зоне $l$, а также критический коэффициент трения между заготовкой и волокой

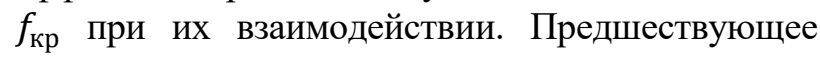
изложение материала по взаимодействию инструмента и заготовки предполагало наличие плавного перехода формы волоки при деформировании заготовки. Такой вид взаимодействия позволил в качестве собственных функций задачи выбрать совокупность синусоидальных кривых, что в расчетной схеме соответствует шарнирному опиранию деформированных слоев заготовки. 
Отметим, что при эксплуатации волочильных установок не исключаются случаи подрезания, шелушения и трещинообразования обрабатываемых изделий [8]. Эти погрешности при обработке имеют место в случаях недостаточной кривизны рабочих поверхностей волокон, назначения высоких скоростей протяжки и высоких степеней обжатия заготовок и деталей. И если же рабочая поверхность волока выполнена по кривой, у которой имеются острые кромки, то возможно «подрезание» поверхностного слоя заготовки и в силу этого деформирование «гипотетической» балки происходит по кривым, близким к косинусоидам. «Гипотетическая» балка может быть уподоблена элементу поверхностного слоя со свободным концом в зоне взаимодействия инструмента и заготовки, и жесткой заделкой в местах затухания деформационной волны по длине заготовки. Длина деформированного участка заготовки подлежит определению, исходя из косинусоидальной формы упругой линии (рис. 5).

Аналогичное проявление процессов подрезания заготовки имеет место при обработке изделий на металлорежущих станках (например, фрезерном или строгальном), что дает нам основание описывать эти разные технологические операции с одних позиций. Ранее нами было принято допущение о том, что поверхностные слои деталей могут быть уподоблены балке на упругом основании, подкрепленной вертикальными связями согласно гипотезе Винклера. Эта гипотеза хорошо согласуется с реальной ситуацией, если отсутствует «подрезание» заготовки и эксцентриситет приложения продольной нагрузки к поверхностным слоям равен 0, при этом схема опирания балки является шарнирной [7].

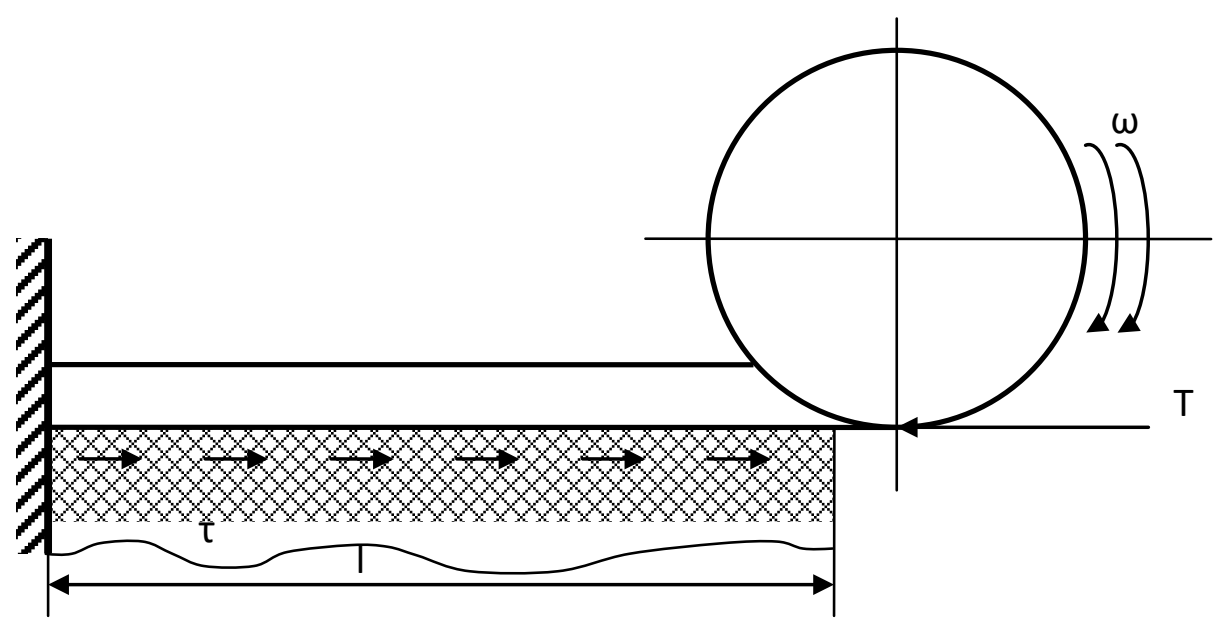

Рис. 5. Кобоснованию возникновения реакций продольных связей при подрезании или резании заготовки: $\tau$ - интенсивность реакций продольных связей; $\mathrm{M}$ - крутящий момент на шпинделе при фрезеровании; $\omega$ - угловая частота вращения шпинделя; $\mathrm{T} \geq \mathrm{T}_{\text {кр }}-$ окружное усилие резания; 1 - длина деформированного участка заготовки

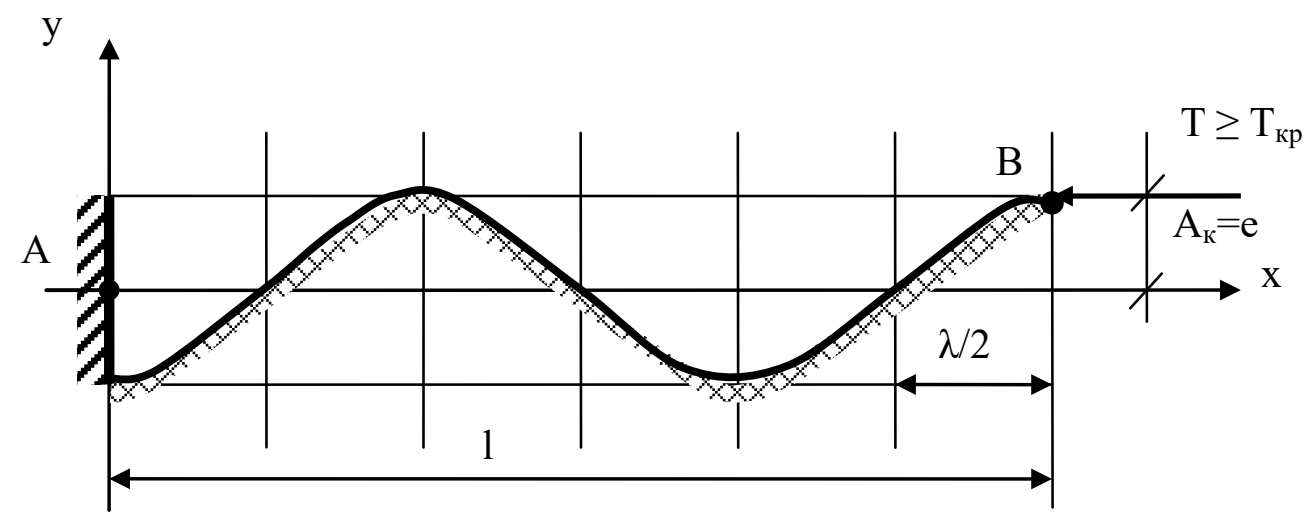

Рис. 6. Схема деформированного участка наружных слоев заготовки (при подрезании): $\mathrm{A}_{\kappa}-$ амплитуда волны деформации (е - эксцентриситет); $\mathrm{C}$ - жесткость продольных связей заготовки; $\beta$ - жескость вертикальных связей заготовки; $\lambda, 1-$ соответственно длина полуволны и длина участка потерявшего устойчивость $(\mathrm{l}=\kappa \lambda)$; к - число полуволн деформации; е - эксцентриситет приложения нагрузки Т; $\mathrm{T}_{\text {кр }}$ - критическая сила 
Если присутствует подрезание поверхностного слоя детали, то расчетная схема балки не может быть принятой шарнирной. Расчетную схему такой балки более правильно принять с одним свободным концом (к которому приложено усилие резания), а вторым - жестко защемленным. Поэтому для принятия достоверной расчетной схемы поверхностных слоев балки необходимо учесть горизонтальные упругие связи балки и их распределенные реакции. Далее рассмотрим расчетную схему балки на упругом основании, которая потеряла упругую устойчивость (рис.5) и её движение в процессе деформирования поверхностных слоев может быть описано дифференциальным уравнением (25) с граничными условиями (52). В этом случае сдвигающая сила от инструмента (фрезы при фрезеровании, резца при поперечном и продольном строгании, острого выступа при волочении и так далее) приводит к динамической потере устойчивости поверхностных слоев и срезающему воздействию этого усилия по плотности (или цилиндрической поверхности) при механической обработке деталей. Отметим, что вертикальные упругие связи «основания» «гипотетической» балки согласно гипотезы Винклера отражены в уравнении (25) и граничных условиях (52). Будем полагать, что горизонтальные связи имеют упруго-фрикционную природу между наружными уплотненными слоями и основанием заготовки при волочении, или между верхними срезаемыми слоями заготовки и основанием обрабатываемой детали при её механической обработке.

Рассматривая взаимодействие между этими слоями при механической обработке равнодействующая касательной сдвигающей силы по отношению к обрабатываемому (срезаемому) слою приложена с эксцентриситетом в связи с тем, что наружные слои при потере устойчивости деформировались и амплитуда волны деформа-

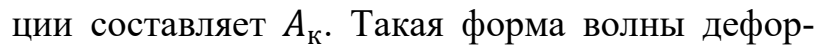
мации свидетельствует о возникновении в очаге разрушения сложного вида деформации и предельного напряженного состояния, которое приводит к появлению первоначальной трещины, тогда трещина под действием предельных уси-

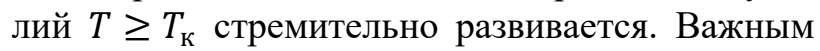
при явлении разрушения является хрупкий или пластический его характер. Так, хрупкое разрушение наступает в случае отрыва элементов обрабатываемого материала [4]. Если же в механизме разрушения объекта преобладает необратимый относительный сдвиг элементов структуры тела, то имеет место пластический характер разрушения (это видно, исходя из характера образовавшейся стружки). «Спусковым механизмом» для разрушения обрабатываемых слоев детали является возникновение волнистой поверхности при потере продольной устойчивости на наружных слоях детали, приводящей сверхкритическим напряжениям, являющихся причиной возникновения первоначальной трещины на контакте изделия и инструмента [4] (рис. 6).

Механизм дальнейшего разрушения поверхностных слоев в случае их механической обработки имеет непрерывный характер до окончания обработки.

Отразим влияние горизонтальных упругих связей в уравнении (25) с учетом граничных и начальных условий (52).

Запишем граничные условия для «гипотетической» балки, считая, что в т. $A$ балка жестко защемлена, а в т. $B$ свободно оперта (поэтому и происходит подрезание и скалывание заготовки):

$$
\begin{aligned}
& w_{x=0}=e ;{\frac{d^{2} w}{d x^{2}}=0}^{=0} ; w(x, t)_{t_{0}}=w_{0}(x) ; \\
& T \frac{d w}{d x}_{x=0}=0 ; Q=T \frac{d w}{d x}_{x=l} ;{\frac{\partial w}{d x} t_{0}}=\frac{\partial w_{0}}{\partial t}(x) .
\end{aligned}
$$

Граничным условиям задачи удовлетворяет функция:

$$
W(x, t)=\sum_{k=0,1,2,3}^{\infty} A_{k}(t) \cos \frac{(2 k+1)}{l} \frac{\pi}{2} x,
$$

которая также удовлетворяет дифференциальному уравнению (25).

Преобразуем дифференциальное уравнение (25) к виду (54), учитывающему горизонтальные упругие связи на границе раздела заготовки на поверхностные и внутренние слои $\Delta T$, окончательно будем иметь:

$$
\begin{gathered}
E_{n} I \frac{\partial^{4} w}{\partial x^{4}}+\left(T_{0}+T_{1 k} \cos p_{k} t+\Delta T\right) \frac{\partial^{2} w}{\partial x^{2}}+ \\
+\beta w+m \frac{\partial^{2} w}{\partial t^{2}}=0 .
\end{gathered}
$$

Для удобства и, не нарушая строгости изложения материала, принимаем, что в этом случае собственные функции и гармонические возмущения во времени изменяются по косинусоидальному закону.

Дифференциальное уравнение (54) отличается от соответствующего уравнения (25) наличием реакции продольных связей в виде

$$
\Delta T=-c v,
$$

где $c$ - жесткость горизонтальной составляющей упругой связи между наружными (обрабатываемыми) и внутренними слоями заготовки, подверженной подрезанию «острой» кромкой воло- 
ки, фрезой или резцом; $v=v(x, t)$ - продольное перемещение точки контакта деформированного слоя, подверженного воздействию инструмента и связанного с поперечным перемещением этого слоя; $T_{1 k}-$ половина амплитуды колебаний нагрузки для одной из собственных частот.

Как следует из [10]:

$$
v=\frac{1}{2} \int_{0}^{l}\left(\frac{d w}{d s}\right)^{2} d s+\frac{1}{8} \int_{0}^{l}\left(\frac{d w}{d s}\right)^{4} d s+
$$

Рассмотрим (53) при $k=0$, тогда

$$
w(x, t)=A_{0}(t) \cos \frac{\pi s}{2 l}
$$

тогда

$$
v=\frac{\pi^{2} A_{0}^{2}}{4 \cdot 4 l}+\frac{3}{64} \frac{\pi^{4} A_{0}^{4}}{4 l^{3}}+\cdots
$$

С учетом реакции упругого основания в горизонтальном направлении (касательное сопротивление материала усилию резания) можно записать уравнение изменения амплитуд колебания во времени (в форме Матье) на основании (53), (54), (56) с учетом продольной просадки «гипотетической» балки.

Рассмотрим уравнение (53) с учетом нулевого приближения, когда $k=0$, тогда

$$
\begin{gathered}
A_{0}^{\|}(t)+2 \varepsilon A_{0}^{\mid}(t)+ \\
+\omega^{2}\left(1-\frac{T_{0}+T_{1} \cos p_{k} t+\Delta T}{T_{\text {кр }}}\right) A_{0}(t)=0 .
\end{gathered}
$$

После подстановки (56) в (53) получим:

$$
\begin{gathered}
A_{0}^{\|}(t)+2 \varepsilon A_{0}^{\mid}(t)+ \\
+\omega^{2}\left(1-\frac{T_{0}+T_{1} \cos p_{k} t}{T_{\text {кр }}}\right) A_{0}(t)+\psi(A)=0,
\end{gathered}
$$

где

$$
\psi(t)=\frac{\pi^{2} \omega^{2} e^{2} A_{0}^{3}}{4 \cdot 4 e T_{\text {кр }}}+\frac{3}{4 \cdot 64} \frac{\pi^{4} \omega^{2}}{e^{3} l^{3} T_{\text {кр }}} \cdot A_{0}^{5}+\cdots
$$

Если нелинейная функция $\psi\left[A_{0}(t)\right]$ определяется в соответствии с выражением (61), то есть она обусловлена нелинейной упругостью, вызванной горизонтальным отпором основания, то для определения амплитуд колебаний достаточно воспользоваться выражением для амплитуд [10] в виде:

$$
A=\frac{2 \Omega}{\sqrt{3 \gamma}} \sqrt{n^{2}-1 \pm \sqrt{\mu^{2}-\frac{n^{2} \Delta^{2}}{\pi^{2}}}}
$$

где $\gamma=\frac{\pi^{2} \omega^{2}}{4 \cdot 8 l^{2}}\left(\frac{2 c l}{T_{\text {кр }}}+1\right)-$ коэффициент нелинейной упругости; $c=G \cdot F=G \cdot b \frac{\lambda}{2}-$ жесткость продольной упругой связи, обусловленная шириной обрабатываемой впадины и длиной одной четверти деформационной волны, расположен- ной в растянутой зоне «гипотетической» балки при потере последней продольной устойчивости; $\omega_{k}=\frac{k^{2} \pi^{2}}{l^{2}} \sqrt{\frac{E I}{m}}-$ значение критической частоты собственных колебаний незагруженного стержня (в силу идентичности определения частот для разных значений $k$, индексы при соответствующих величинах опускаются без нарушения общности рассуждений); $\Omega_{k}=$ $\omega_{k} \sqrt{1-\frac{T_{0}}{T_{\text {кр}}}}-$ частота собственных колебаний стержня, загруженных постоянной составляющей продольной силы; $\mu_{k}=\frac{T_{t}}{2\left(T_{\mathrm{\kappa p}}^{k}-T_{0}\right)}-$ малый параметр, называемый коэффициентом возбуждения нагрузки для частоты собственных колебаний нагрузки с индексом $k ; n=\frac{\theta}{2 \Omega}-$ коэффициент, описывающий отношение частоты возбуждения колебаний к частоте собственных колебаний стержня, загруженного постоянной силой (при главном резонансе $n \approx 1$ ); $\Delta=\frac{2 \pi \varepsilon}{\Omega}-$ декремент затухания колебаний в системе (при линейном затухании).

Для удобства использования уравнения (60) и построения его приближенного решения относительно изменяющихся амплитуд колебаний $A_{0}(t)$ запишем его в виде:

$$
\begin{gathered}
A_{0}^{\|}(t)+2 \varepsilon A_{0}^{\mid}(t)+\Omega^{2}(1-2 \mu \cos \theta t) A_{0}+ \\
+\psi\left(A_{0}(t)\right)=0 .
\end{gathered}
$$

Учитывая слабую нелинейность (63) подставим $w(x, t)$ при $[t=c o n s t]$ в линеаризованную часть (54). Из найденных результатов получим формулу для критической силы $T_{\text {кр}}$, числа полуволн деформации на длине балки $l$ и значение длины участка балки, потерявшей устойчивость.

Воспользовавшись формулой амплитуд установившихся колебаний (62) или решением уравнения (63), получим результат, который зависит от особенностей нагружения обрабатываемой детали формообразующим (или металлообрабатывающим) инструментом, а также от физико-механических свойств взаимодействующих элементов процесса. Вместе с тем, при металлообработке, в большинстве случаев, величина установившейся амплитуды колебаний зависит от расположения начальной неправильности волоки, или глубины подачи фрезы или резца, то есть величину $A_{0}$ можно считать заданной как граничное условие на свободном конце слоя при стационарном его рассмотрении.

Обратимся к случаю взаимодействия инструмента и детали при её подрезании. При этом 
нарушаются условия обтекания инструмента (в первом приближении «штампа») деформируемым материалом и блокировка его обтекания вызывает нарушение сплошности материала [9]. Кроме того, наблюдается царапание (микрорезание или раздир обрабатываемой поверхности как на микро так и макроуровнях). Для этого случая число циклов нагружения составляет $n=1$, при слабой смазке (рис. 4). Переходя к силовому взаимодействию заготовки и инстру-

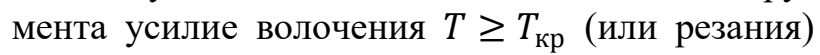
будет превышать неизвестное критическое усилие и будет приложено к поверхностным слоям заготовки с эксцентриситетом и численно равно глубине подачи инструмента в заготовку.

Исходя из характера деформации наружных слоев, примем допущение о том, что эта волна имеет периодический характер с переменной амплитудой, и в начале процесса внедрения инструмента принимает значение $A_{k}=e$. Кроме того, точка приложения касательного усилия будет удалена от вершины деформированной поверхности заготовки к её внутренним слоям на величину $e$ (рис. 6). Так как участок «гипотетической» балки длиной $\lambda / 2$, расположенный слева от точки $B$ (рис. 6), находится под действием изгибающего момента и нормальных напряжений, то естественно предположить, что первоначальный излом наружного слоя произойдет левее точки $B$, и по мере дальнейшего внедрения инструмента в деталь, зона излома и скола материала будет перемещаться к началу системы координат, при этом касательное и нормальное напряжения на длине участка балки $\lambda / 2$ будут равны:

$$
\begin{gathered}
\tau_{\text {max }} \geq \tau_{\text {кр }}=\frac{3}{2} \frac{\tau_{\text {кр }}}{b l} ; \\
\sigma_{\text {max }} \geq \sigma_{\text {кр }}=\frac{T_{\mathrm{\kappa p}} \lambda}{2 b l^{2}} .
\end{gathered}
$$

В приведенных выражениях (64) и (65) величина $b$ определяется шириной инструмента, а $T_{\text {кр }}, l, \lambda$ - неизвестные величины соответственно критической сжимающей силы, длина балки, потерявшей упругую устойчивость, и длина одной полуволны балки, потерявшей устойчивость. Указанные величины подлежат определению, так как изменился вид исходного дифференциального уравнения вместо (25) формула (54), а также изменился вид граничного условия (вместо (28) будут формулы (52)).

Поэтому механика взаимодействия инструмента и заготовки для случаев шарнирного опирания концов и свободно опертого наружного слоя (в одной точке и защемленного в другой) будут различными.

Как следует из расчетной схемы задачи, продольная сила $T$ (рис. 5) вызывает продольный изгиб поверхностных слоев по кривым, близким к косинусоидам, при этом первая четверть волны деформируемой балки будет находиться в зоне действия растягивающих напряжений, а вторая и третья четверти длины балки будут находится в зоне действия сжимающих напряжений. В этом случае усилие резания прикладывается к заготовке в зоне первой четверти волны деформации, производя срез или хрупкий скол в пределах длины и глубины разрушаемого слоя. Отметим, что в процессе разрушения значительная часть усилия резания будет передаваться по плоскости раздела слоев, параллельно горизонтальной оси, разрушая продольные связи между поверхностными и внутренними слоями детали или заготовки. Так как в пределах первой четверти усилие $T$ прилагается к наружным слоям консольно и с эксцентриситетом, то эта часть балки (длиной $\lambda / 2$ ) находится под воздействием изгибающих напряжений, последующая же часть балки находится в зоне сжатия, поэтому эксцентрично приложенная сила $T$ стремится вызвать излом или чешуйчатое разрушение материала в пределах первой четверти деформированных поверхностных слоев балки (длиной $\lambda / 2$ ).

По мере осуществления процесса механической обработки, зона разрушения продвигается к конечной точке обработки (со скоростью процесса $v_{n}$ ). При этом бегущая волна деформации опережает инструмент (случай подвижного инструмента) для строгания и фрезерования, или отстает от инструмента (неподвижный инструмент и «стоячая» волна деформации для волочения на величину $0<x<l$, где $x$ - текущая координата) при прохождении волны деформации длиной $l$ по заготовке или детали.

Неизвестные параметры деформационной волны на обрабатываемом участке с одним свободным концом, а вторым - жестко защемленным, согласно граничных условий (52) найдем по аналогии с граничными условиями задачи (28) для случая шарнирного опирания концов.

В этом случае для определения величины

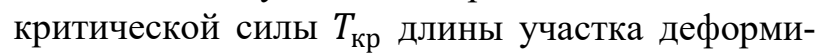
рованного слоя $l$ и число полуволн $k$ будем полагать, что $A_{k}$ не зависит от времени и при этом достигает амплитудных значений. Тогда, подставляя выражение (53) в дифференциальное уравнение (25), получим: 


$$
\begin{gathered}
\frac{(2 k+1)^{4}}{l^{4}}\left(\frac{\pi}{2}\right)^{4} A_{k} E_{n} I-T_{\text {кр }} A_{k} \frac{(2 k+1)^{2}}{l^{2}}\left(\frac{\pi}{2}\right)^{2}+ \\
+\beta A_{k}=0 \\
T_{\text {кр }}=\frac{(2 k+1)^{4}}{l^{4}}\left(\frac{\pi}{2}\right)^{4} E_{n} I+\beta \frac{l^{2}}{(2 k+1)^{2}}\left(\frac{2}{\pi}\right)^{2} .
\end{gathered}
$$

Если учесть зависимость между вертикальной жесткостью основания и модулем упругости внутренних слоев заготовки, то получим согласно [6] в виде $\beta=\frac{E}{2} \sqrt[3]{\frac{E}{4 I E_{c}}}$,

$$
T_{\text {кр }}=\frac{(2 k+1)^{2}}{l^{2}}\left(\frac{\pi}{2}\right)^{2} E_{n} I+\frac{E}{2} \sqrt[3]{\frac{E}{4 E_{c} I}} \frac{l^{2}}{(2 k+1)^{2}} \frac{2}{\pi}^{2}
$$

или

$$
T_{\text {кр }}=\frac{(2 k+1)^{2}}{l^{2}}\left(\frac{\pi}{2}\right)^{2} E_{n} I+\frac{E}{2} \frac{2 l}{(2 k+1) \pi} .
$$

Введем обозначение

$$
\frac{(2 k+1)}{l} \frac{\pi}{2}=\alpha
$$

откуда выразим $k$ - неизвестное число полуволн на участке условной балки $l$, потерявшей упругую устойчивость:

$$
k=\frac{\alpha l}{\pi}-\frac{1}{2}
$$

Исходя из физических соображений [6] запишем выражение для $\alpha$ в виде:

$$
\alpha=\sqrt[3]{\frac{E}{4 E_{n} I}},
$$

где $\alpha$ - трактуется как неизвестное число полуволн на участке балки длиной $\pi$.

Тогда, с учетом (71) неизвестное число полуволн на участке балки $l$, потерявшей упругую устойчивость будет равно:

$$
k=\frac{l}{\pi} \sqrt[3]{\frac{E}{4 E_{n} I}}-\frac{1}{2} .
$$

Для балки на упругом основании число полуволн $k$ находим из условия минимума нагрузки.

Учитывая тот факт, что правый конец балки, взаимодействующий с волокой, принят, как свободный, лежащий (свободно) на упругом основании, то изогнутая линия балки будет представлена в виде косинусоиды с количеством полуволн $k$ (рис. 6). Упругая линия балки связана с дискретной функцией от $k$ и $\alpha$. Переход от ветви косинусоиды с $(2 k+1)$ полуволнами к косинусоиде с $(2 k+2)$ полуволнами, по аналогии с работой [7], может быть осуществлена, исходя из соотношения:

$$
\frac{(2 k+1)^{2}}{l^{2}}\left(\frac{\pi}{2}\right)^{2} E_{n} I+\beta \frac{l^{2}}{(2 k+1)^{2}} \frac{2}{\pi}^{2}=
$$

$$
=\frac{(2 k+2)^{2}}{l^{2}}\left(\frac{\pi}{2}\right)^{2} E_{n} I+\beta \frac{l^{2}}{(2 k+2)^{2}}\left(\frac{2}{\pi}\right)^{2} .
$$

Рассмотрим левую часть (73):

$$
\begin{gathered}
-\left(4 k^{2}+4 k+1\right) \frac{\pi^{2}}{4} \frac{E_{n} I}{l^{2}}+\left(4 k^{2}+8 k+4\right) \frac{\pi}{4} \frac{E_{n} I}{l^{2}}= \\
=(4 k+3) \frac{\pi^{2}}{4} \frac{E_{n} I}{l^{2}} .
\end{gathered}
$$

Обратимся снова к (72):

$$
\begin{gathered}
(4 k+3) \frac{\pi^{2}}{4} \frac{E_{n} I}{l^{2}}= \\
=\beta l^{2}\left(\frac{2}{\pi}\right)^{2}\left[\frac{1}{(2 k+1)^{2}}-\frac{1}{(2 k+2)^{2}}\right]
\end{gathered}
$$

или

$$
\begin{gathered}
(4 k+3) \frac{\pi^{2}}{4} \frac{E_{n} I}{l^{2}}= \\
=\beta l^{2}\left(\frac{2}{\pi}\right)^{2}\left[\frac{4 k^{2}+8 k+4-4 k^{2}-4 k-1}{(2 k+1)^{2}(2 k+2)^{2}}\right], \\
\beta l^{2} \frac{4}{\pi^{2}}=\frac{\pi^{2}}{4} \frac{E_{n} I}{l^{2}}\left[(2 k+1)^{2}(2 k+2)^{2}\right],
\end{gathered}
$$

или

$$
\frac{\beta}{E_{n}}=\frac{\pi^{4}}{l^{4}}\left[(2 k+1)^{2}(2 k+2)^{2}\right] .
$$

Для определения числа полуволн $k$ на деформированной части $l$ необходимо воспользоваться выражениями (73) и (77). Обе величины остаются пока неизвестными. Однако, полученное выражение для $k$ и $l$ прямо не содержит кри-

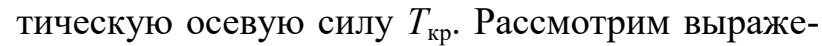
ние для критической силы балки на упругом основании, считая, что прогибы балки изменяются по косинусоидальному закону. Эта задача схожа c задачей о подрезании твердым индентором наружного слоя заготовки (из мягких материалов, и скола наружных слоев для хрупких материалов в рамках задачи о потере балкой на упругом основании продольной устойчивости). Под твердым индентором будем понимать, как режущий инструмент, так и любую выступающую микронеровность инструмента.

Свяжем количество полуволн на деформированной части балки через критическую силу с числом полуволн деформации из условия её минимума. Так как переменная $\frac{(2 k+1)}{l} \frac{\pi}{2}$ является дискретной, то запишем выражение критической силы для $\frac{(2 k+1)}{l}$ полуволн и с учетом перескока её упругой линии на форму с $\frac{(2 k+2)}{l}$ полуволнами:

$$
\begin{aligned}
& \frac{(2 k+1)^{2}}{l^{2}} \frac{\pi^{2}}{4}+\frac{\beta}{E_{n} I} \frac{l^{2}}{(2 k+1)^{2}}\left(\frac{4}{\pi^{2}}\right)= \\
& =\frac{(2 k+2)^{2}}{l^{2}} \frac{\pi^{2}}{4}+\frac{\beta}{E_{n} I} \frac{l^{2}}{(2 k+2)^{2}}\left(\frac{4}{\pi^{2}}\right) .
\end{aligned}
$$


Предварительно преобразуем выражение, содержащее слагаемое с вертикальной жесткостью упругого основания $\beta$ :

$$
\begin{gathered}
\frac{\beta l^{2}}{E_{n} I}\left(\frac{4}{\pi^{2}}\right)\left[\frac{1}{(2 k+1)^{2}}-\frac{1}{(2 k+2)^{2}}\right]= \\
=\frac{\beta l^{2}}{E_{n} I}\left(\frac{4}{\pi^{2}}\right)\left[\frac{(2 k+2)^{2}-(2 k+1)^{2}}{(2 k+1)^{2}(2 k+2)^{2}}\right]= \\
=\frac{\beta l^{2}}{E_{n} I}\left(\frac{4}{\pi^{2}}\right)\left[\frac{4 k^{2}+8 k+4-4 k^{2}-4 k-1}{(2 k+1)^{2}(2 k+2)^{2}}\right]= \\
=\frac{\beta l^{2}}{E_{n} I}\left(\frac{4}{\pi^{2}}\right) \frac{4 k+3}{(2 k+1)^{2}(2 k+2)^{2}} .
\end{gathered}
$$

Тогда выражение, связанное с кривизной балки, имеет вид:

$$
\begin{gathered}
\frac{\pi^{2}}{4} \frac{4 k^{2}+8 k+4-4 k^{2}-4 k-1}{l^{2}}= \\
=\frac{\pi^{2}}{4 l^{2}}(4 k+3) .
\end{gathered}
$$

Из выражений (77) и (78) находим:

$$
\frac{\pi^{2}}{4 l^{2}}(4 k+3)=\frac{\beta l^{2}}{E_{n} I}\left(\frac{4}{\pi^{2}}\right) \frac{(4 k+3)}{(2 k+1)^{2}(2 k+2)^{2}}
$$

Тогда:

$$
\frac{E_{n} I}{\beta} \frac{\pi^{4}}{16}\left[(2 k+1)^{2}(2 k+2)^{2}\right]=l^{4} .
$$

В дальнейшем учтем выражения $\beta=\frac{E}{2} \alpha$; $2 k+1=\frac{\alpha}{2 \pi} ; \alpha=\sqrt[3]{\frac{E}{4 E_{n} I}} ; 2 k+2=\frac{\alpha l}{2 \pi}+1$, тогда получим квадратное уравнение для определения $l$ - длины участка обрабатываемой заготовки, потерявшей продольную устойчивость:

$$
\begin{gathered}
\frac{E_{n} I}{\beta} \frac{\pi^{4}}{16} \frac{\alpha^{2}}{4 \pi^{2}}\left(\frac{\alpha^{2} l^{2}}{4 \pi^{2}}+\frac{\alpha l}{\pi}+1\right)=l^{2} ; \\
(4 k+3) \frac{\pi^{2}}{4} \frac{E_{n} I}{l^{2}}=\beta l^{2}\left(\frac{2}{\pi}\right)^{2}\left[\frac{1}{(2 k+1)^{2}}-\frac{1}{(2 k+2)^{2}}\right] ; \\
l^{2}+\frac{\frac{E_{n} I \pi}{\beta 64} \alpha^{3}}{\frac{E_{n} I \pi^{2}}{\beta 256} \alpha^{4}-1} l+\frac{\frac{E_{n} I \pi^{2}}{\beta} \alpha^{2}}{\frac{E_{n} I \pi^{2}}{\beta 256} \alpha^{4}-1}=0,
\end{gathered}
$$

тогда

$$
l_{1,2}=\sqrt[3]{\frac{4 E_{n} I}{E}}\left[\frac{\pi}{256} \pm \sqrt{\frac{\frac{\pi^{2}}{256^{2}}}{\left(1-\frac{\pi^{2}}{512}\right)^{2}}+\frac{\frac{\pi}{128}}{\left(1-\frac{\pi^{2}}{512}\right)}}\right],
$$

где $l_{1,2}$ - длина балки, потерявшей упругую устойчивость.

Таким образом, получено квадратное уравнение для определения длины участка заготовки, потерявшей продольную устойчивость через модули упругости обрабатываемого наружного слоя и сердцевины заготовки, а также предела текучести материала заготовки и числа полуволн деформации $k$ через $\alpha$ [см. формулы (44), (45), (47)].
После определения значения критической силы $T_{\text {кр }}$, которая может быть легко реализована в реальном процессе и её приложение к заготовке при недостаточно обоснованной геометрии волоки (её деформирующей части), происходит потеря устойчивости наружных слоев, напоминающая срез или скол поверхностного слоя, который будет происходить по одной или нескольким полуволнам наружного слоя, толщина которого равна полуразности наружных размеров заготовки и калибрующего размера волоки и может быть приравнена к амплитуде деформации полуволны $A_{\mathrm{k}}$. Аналогичные эффекты проявляются при механической обработке деталей и заготовок.

В конечном счете, нами получены параметры деформационных волн при механической обработке и обработке металлов давлением. Проявление этих деформационных процессов затрудняет определение оптимальных условий выполнения механической обработки на металлорежущих станках и волочильных установках. Как было нами показано, рассматриваемые технологические операции напрямую связаны с характеристиками трения между заготовкой и инструментом, особенно в зоне минимальных значений сил трения, как функций от скорости процесса механической обработки или скорости проскальзывания детали по инструменту при выполнении рабочих процессов. Поэтому нас будут интересовать скоростные режимы, приводящие к минимальным значениям сил трения, а также определенные физические воздействия на контактирующие детали и инструменты.

Перейдем к определению условий оптимального протекания или интенсификации рассматриваемых рабочих процессов. В этой связи отметим, что в промышленности существуют вибрационные методы, которые позволяют путем наложения вынужденных вибраций оптимизировать процессы механической обработки заготовок и деталей по нескольким параметрам $[11,12,13]$.

Выводы и задачи дальнейших исследований. Из анализа фрикционных и вызванных ими параметрических колебаний следует вывод, что эти самовозбуждающиеся колебания при обработке металлов давлением и резании металлов на металлорежущих станках, во многих случаях приводит к неустойчивым технологическим процессам, которые не всегда обеспечивают высокое качество готовых изделий. Эти нежелательные проявления объясняются «ниспадающим» характером силы трения между инстру- 
ментом и деталью при изменении скорости их взаимного проскальзывания.

Технологические особенности процессов резания и волочения в определенных пределах позволяют добиться оптимального стружкообразования (и в ряде случаев стружкодробления), а также достаточно высокого качества обрабатываемой поверхности при обработке металлов давлением (и волочении). Так, например, для получения заданной формы стружки необходимо изготовить ряд дополнительных устройств, обеспечивающих её естественное дробление [13]. Однако, этот подход не является приоритетным.

Поэтому для интенсификации технологических процессов по обработке металлов давлением и процессов резания необходимо применить метод, основанный на гарантированном снижении усилий и коэффициентов трения при соответствующем способе механической обработки деталей и заготовок (рис. 7, 8, 9, 10).

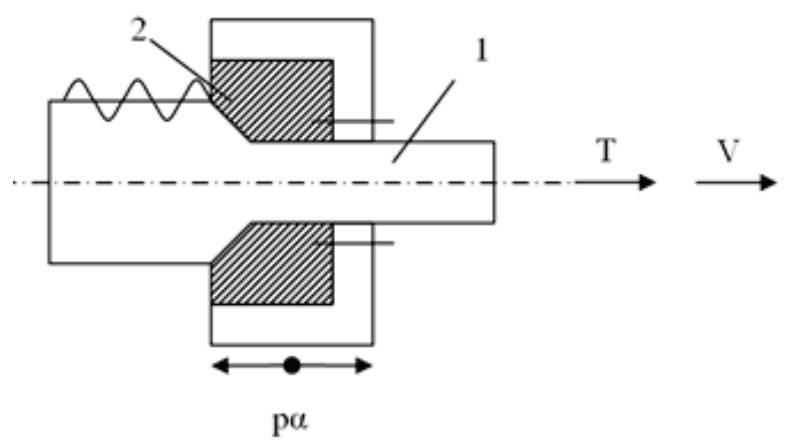

Рис. 7. Вибрационное волочение проволоки (японский патент № 289832): 1 - проволока; 2 - валок; $\tilde{p}, \alpha$ - частота и амплитуды вибрации; $V$ - скорость волочения; $T$ усилие волочения.

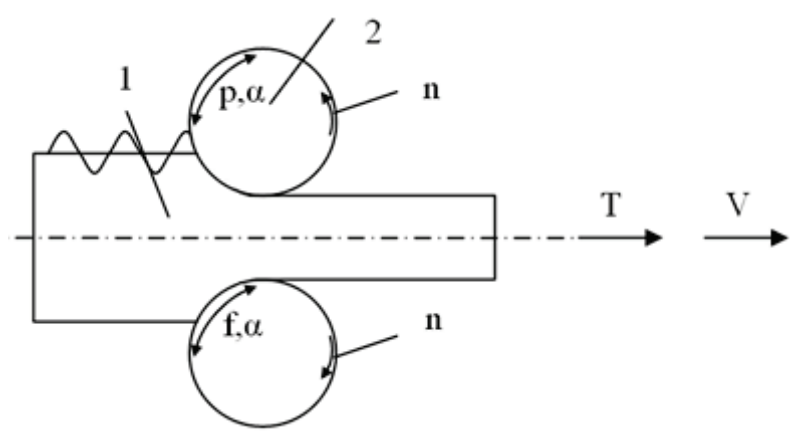

Рис. 8. Схема вибрационной прокатки: 1 - заготовка; 2 - валок; $n$ - угловая частота вращения валков; $\tilde{p}, \alpha$ - частота и амплитуда вибрации; $V$ - скорость прокатки; $T-$ усилие прокатки.

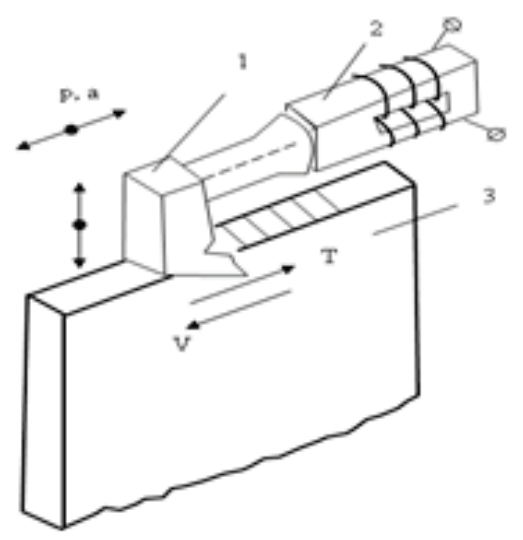

Рис. 9. Схема вибрационного строгания: 1 - резец; 2 - вибратор с концентратором; 3 - деталь; $V$ - скорость строгания; $\tilde{p}$, $a$ - частота и амплитуда вибрации при горизонтальных (или вертикальных) вибрациях; $T$ - усилие резания

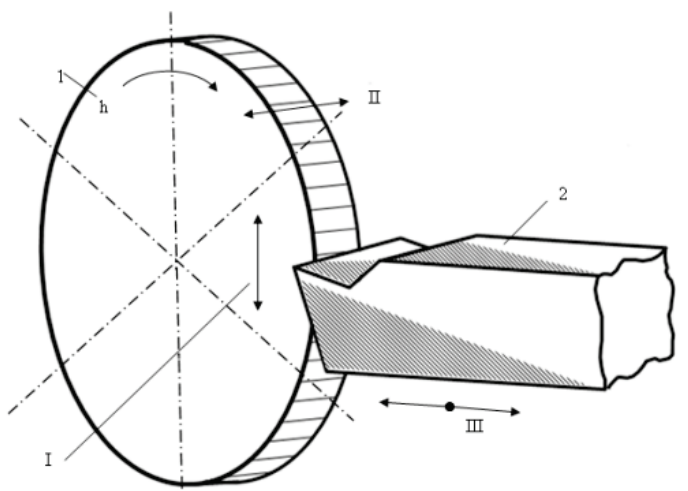

Рис. 10. Вибрационное точение: 1 - деталь; 2 - резец; I, II, III - направления приложенных вибраций; $n$ - угловая частота вращения шпинделя

В ряде случаев для улучшения параметров механической обработки изделий является эффективным применение технологических процессов с направленными вибрациями $[11,12$, 13]. Особенно ощутимым является наложение вибраций при обработке труднообрабатываемых материалов (рис. 9, 10). Следуя рекомендациям $[11,12]$ необходимо стремиться к применению генераторов колебаний с весьма малыми амплитудами колебаний в пределах $10 \ldots 20$ мк, при этом линейная скорость механической обработки должна быть связана с амплитудой $T_{1 \mathrm{k}}$ и частотой наложенных вибраций неравенством:

$$
v_{n}<2 \pi T_{1 k} \widetilde{\theta_{k}}
$$

где $v_{n}$ - линейная скорость рассматриваемого технологического процесса, а $T_{1 k}$ и $\theta_{k}$ - соответственно амплитуда и частота наложения вибраций. 
В силу неравенства (82) частота виброперемещений должна быть значительной, порядка 100 кГц и выше. Кроме того, частота вибровозбуждения должна, по возможности, быть близкой к одной из высших собственных частот в системе инструмент-суппорт относительно обрабатываемой детали, или, как вариант, близкой к одной из высших собственных частот системы деталь-станок: $\widetilde{\theta_{k}} \leq \widetilde{P_{k}}$, где $\widetilde{\theta_{k}}-$ частота колебаний, излучаемых вибратором (величина регулируемая); $\widetilde{P_{k}}-$ одна из высших собственных частот колебаний в системе инструмент-суппорт.

Исходя из сказанного следует, что вынужденные колебания поверхностных слоев детали с налаженными вибрациями при волочении, фрезеровании, строгании и других аналогичных операциях могут быть описаны одним из дифференциальных уравнений (63) для собственной формы с индексом $k$, частотой возбуждения $\widetilde{P_{k}}$ и амплитудой виброперемещений $T_{1 k}$.

Запишем дифференциальное уравнение вынужденных колебаний поверхностных слоев заготовки с наложенными вибрациями (от внешнего вибратора), которое по форме совпадает с дифференциальными уравнениями (54) или (25).

$$
\begin{aligned}
E_{n} I \frac{\partial^{4} w}{\partial x^{4}}+ & \left(T_{0}+T_{1 k} \cos \theta_{k} t+\Delta T\right) \frac{\partial^{2} w}{\partial x^{2}}+ \\
& +\beta w+m \frac{\partial^{2} w}{\partial x^{2}}=0,
\end{aligned}
$$

где $T_{1 k}$ - половина амплитуды вынужденных колебаний инструмента, соответствующая частоте вынужденных колебаний $\theta_{k}$ вибратора; $k-$ индекс одной из собственных частот колебаний инструмента $P_{k}$.

Причем $\theta_{k} \leq P_{k}\left(\theta_{k} \approx P_{k}\right)$.

Остальные обозначения соответствуют обозначениям (25), (54).

Для определения амплитуд вынужденных колебаний заготовки используем выражение (62) амплитуд или дифференциальное уравнение (63) с индексом $k$, который определяется экспериментально, таким образом, чтобы выполнялось условие (82).

При этом следует иметь в виду, что уравнения, описывающие наведенные вибрационные процессы включают величины $\theta_{k}$ и $T_{1 k}$, которые зависят только от параметров вибратора, а уравнение (83), которое по форме совпадает с уравнением (54), напрямую связано с характеристикой трения между деталью и инструментом и по сути является уравнением фрикционных автоколебаний с неустойчивыми параметрами. Как следует из [2], причиной возникновения автоколебаний при некоторых режимах резания и об- работки металлов давлением является «ниспадающий» характер силы трения от скорости протекания процесса обработки (или скорости проскальзывания) между инструментом и деталью. Если рассматривать процесс трения детали и инструмента на микроуровне, как явление схватывания соприкасающихся микронеровностей, то изменение силы трения при взаимодействии поверхностей можно трактовать как разрушение «гипотетических» мостиков сварки между контактирующими микронеровностями [9].

Используя обе концепции возникновения нестационарных режимов трения будем полагать, что наложенные высокочастотные вибрации в силу высоких скоростей при виброперемещениях в определенные моменты времени могут существенно повысить скорости взаимного проскальзывания взаимодействующих элементов, а также при взаимодействии микронеровностей с высокими вибрационными скоростями за счет диссипации разогревать поверхностные слои и легко срезать ставшими податливыми мостики «сварки».

Заметим, что диссипация энергии трения за счет высокотемпературных вспышек между микронеровностями приводит к их пластическому состоянию с к последующему разрушению при взаимодействии фрикционных пар.

Такой подход позволяет обосновать основные преимущества вибрационного резания, которые сводятся, в основном, к снижению режущих усилий (или усилий протягивания), улучшению качества обрабатываемых поверхностей (в ряде случаев появляется возможность исключить финишные операции).

Причем преимущества вибрационной мехобработки будут более ощутимы, если помимо выполнения условия (82), вибрационное воздействие будет полностью восприниматься деталью (рис. 9, 10), и не будет передаваться на конструкцию обрабатывающей машины. Такое воздействие становится возможным, если наложенные вибрации по частоте не превысят близкую собственную частоту системе инструмент с суппортом относительно обрабатываемой детали [12] (или волоки с оправкой относительно заготовки). Однако в этом случае можно не получить желаемого результата из-за низкой вибрационной скорости, так как при относительно низких частотах наложения вибраций может не выполняться условие (82).

Таким образом, при вибрационном резании импульсное усилие контакта резца с деталью невелико, в этих условиях силовые воздействия сосредотачиваются в области взаимодействия 
резца с деталью, и динамическая реакция возникает лишь в местах удара. Следовательно, при вибрационной обработке для получения требуемой формы и качества изделий требуется применение незначительной импульсной силы [11, 12]. В этой связи импульсная обработка позволяет эффективно использовать ограниченное количество энергии. Заметим, что наложенные вибрации могут быть как синфазными, так и противофазными по отношению к самовозбуждающимися колебаниям соответственной частоты.

Кроме того, наложенные противофазные вынужденные колебания при выраженном автоколебательном фоне, сопровождающем основной процесс резания, может привести к промежуточному эффекту, который сводится к значительному снижению самовозбуждающихся колебаний в системе «деталь-инструмент-станок», и как результат - повышению качества обрабатываемых поверхностей.

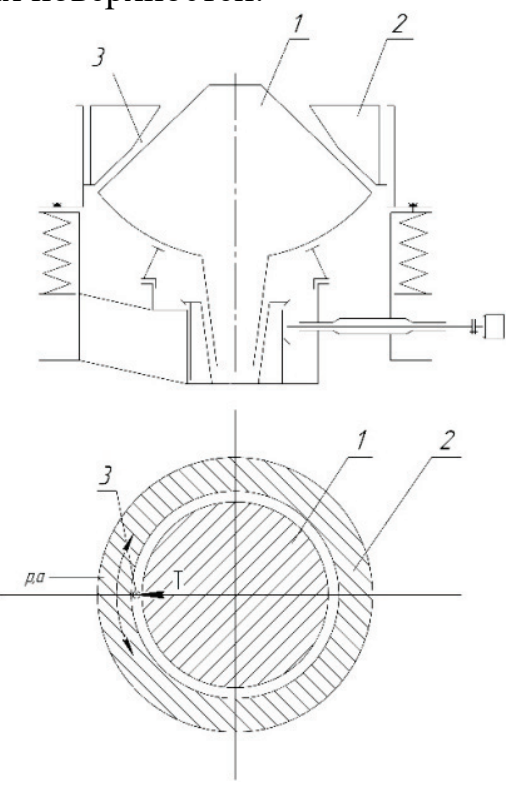

Рис. 10. Конусная дробилка с вибрационным воздействием: 1 - подвижный конус; 2 - неподвижная бронь; 3 - дробимый материал; $\rho, a$ - частота и амплитуда вынужденных колебаний с касательным воздействием; Т - сжимающие силы

На основании изложенного следует вывод, что импульсные силовые воздействия могут быть использованы при различных видах обработки металлов давлением, таких, как штамповка, холодная и горячая прокатка, волочение, обкатка, трубопрокатка, вытяжка, чеканка, гибка (рис. 7, 8).

Другим важным направлением использования импульсного силового воздействия является механическая обработка изделий при точении, сверлении, фрезеровании, нарезании резьб, нарезании зубчатых колес, обработка абразивными частицами и других технологических операциях (рис. 5, 9, 10).

Также метод может быть рекомендован к применению при совершенствовании процессов бурения, дробления и измельчения горных пород и других процессов (рис. 11).

\section{Библиографический список}

1. Смирнов В. В., Яковлев Р.А. Механика приводов прокатных станов. Москва: Металлургия, 1977.

2. Пановко Я. Г., Губанова И. И. Устойчивость и колебания упругих систем. Москва : Наука, 1967.

3. Параметрические эффекты при фрикционном взаимодействии инструмента с заготовкой в процессе её обработки давлением/А. Д. Учитель и др. Металлургическая $u$ горнорудная промьиленность. 2019. № 1-2. C. $17-30$.

4. Сопротивление материалов / А. Ф. Смирнов и др. Москва : Высшая школа. 1969.

5. Светлицкий В. А., Стасенко И. В. Сборник задач по теории колебаний. Москва: Высшая школа, 1979.

6. Волнообразование на обкатываемых поверхностях / Л. Б. Эрлих и др. Москва : Наука, 1973.

7. Вольмир А. С. Устойчивость деформируемых систем. Москва : Наука, 1972.

8. Проскуряков Ю. Г. Упрочняющекалибрующие методы обработки : справочное пособие. Москва : Машиностроение, 1965.

9. Основы расчета на трение и износ/ И. В. Крагельский и др. Москва : Машиностроение, 1977.

10. Болотин В. В. Динамическая устойчивость упругих систем. Москва : ГИТТЛ, 1956.

11. Кумабе Д. Вибрационное резание. Москва : Машиностроение, 1980.

12. Марков А. И. Ультразвуковое резание труднообрабатываемых материалов. Москва : Машиностроение, 1968.

13. Подураев В. Н. Обработка резанием с вибрациями. Москва : Машиностроение, 1970.

\section{References}

1. Smirnov, V. V., \& Iakovlev, R. A. (1977). Mekhanika privodov prokatnykh stanov. Moskva: Metallurgiia.

2. Panovko, Ia. G., \& Gubanova, I. I. (1967). Ustoichivost i kolebaniia uprugikh sistem. Moskva: Nauka. 
3. Uchitel, A. D. et al. (2019). Parametricheskie effekty pri friktsionnom vzaimodeistvii instrumenta s zagotovkoi v protsesse ee obrabotki davleniem. Metallurgicheskaia i gornorudnaia promyshlennost, (1-2), 17-30.

4. Smirnov, A. F. et al. (1969). Soprotivlenie materialov. Moskva: Vysshaia shkola.

5. Svetlitskii, V. A., \& Stasenko, I. V. (1979). Sbornik zadach po teorii kolebanii. Moskva: Vysshaia shkola.

6. Erlikh, L. B. et al. (1973). Volnoobrazovanie na obkatyvaemykh poverkhnostiakh. Moskva: Nauka.

7. Volmir, A. S. (1972). Ustoichivost deformiruemykh sistem. Moskva: Nauka.

8. Proskuriakov, Iu. G. (1965). Uprochniaiushche-kalibruiushchie metody obrabotki. Moskva: Mashinostroenie.

9. Kragelskii, I. V. et al. (1977). Osnovy rascheta na trenie i iznos. Moskva: Mashinostroenie.

10. Bolotin, V. V. (1956). Dinamicheskaia ustoichivost uprugikh sistem. Moskva: GITTL.

11. Kumabe, D. (1980). Vibratsionnoe rezanie. Moskva: Mashinostroenie.

12. Markov, A. I. (1968). Ultrazvukovoe rezanie trudnoobrabatyvaemykh materialov. Moskva: Mashinostroenie.

13. Poduraev, V. N. (1970). Obrabotka rezaniem s vibratsiiami. Moskva: Mashinostroenie.

Мета: досліджувати динаміку взаємодіі поверхневих шарів заготівки з інструментом при виконанні високоточних і енергоємних технологічних операцій по обробиі металів тиском $i$ механічної обробки різанням, а також виконати пошук методів інтенсифікаџії вказаних робочих прочесів, що дозволяють підвищити якість готових виробів $i$ знизити енергоємність технологічних процесів.

Методика досліджень. Для досліджень прийнята модель, в якій інструмент умовно представлений у вигляді жорсткого штампу, а деталь представлена у вигляді податливої плоскої смуги за різних умов того, що спирається зовнішніх шарів оброблюваних виробів.

У роботі використана гіпотеза про те, щсо нелінійне тертя є джерелом коливань, що самозбуджуються, між інструментом і деталлю, які призводять до виникнення завищених зусиль різання $і$ отримання оброблених поверхонь, у ряді випадків, не досить високої якості.

Побудовані математичні моделі для опису деформованих поверхневих шарів деталі під впливом критичних навантажень.
Рекомендовані методи підвищення ефективності обробки виробів, які зводяться до застосування накладення вібрацій з певними амплітудами $і$ частотами, причому, дія цих вібрацій зводиться до виводу і рішення рівнянь, співпадаючих за формою з рівняннями для що самозбуджується коливань.

Результати. У роботі поставлено і вирішено завдання дослідження механіки взаємодіі поверхневих шарів заготівлі з інструментом при виконанні різних технологічних операцій 3 урахуванням характеристики тертя між контактуючими елементами. Для сформульованих завдань на оброблюваній поверхні виникає залишкова хвиля, яка при подальшій обробиі частково згладжується різальним інструментом.

Авторами, для даних видів технологічних операчій визначені довжина залишкової деформаційної хвилі, кількість гребенів на ціий хвилі, а також значення критичної сили, що викликає цุю хвилю.

Наукова новизна полягає в тому, що для опису прочесу волочіння на волочильних установках і проиесу механічної обробки виробів на металорізальних верстатах застосована одна $i$ та ж розрахункова модель $i$ використаний один і той же математичний апарат. Для інтенсифікації даних технологічних процесів рекомендовано використовувати накладення вібрачії з оптимальним налаштуванням по частоті і амплітудi.

Практична цінність роботи полягає в тому, що вказаний метод інтенсифікаиії робочих прочесів при оптимальному виборі параметрів накладених вібрацій може бути успішно застосований при волочінні холодному $i$ гарячому плющенню, штампуванню, обробиі фрезеруванням, струганням, точінням, дробленням подрібненням $і$ бурінням гірських порід і інших технологічних прочесів.

Ключові слова: волочіння, плющення, накатка, штампування, заготівля, деталь, фрезерування, стругання, характеристика тертя, деформачійна хвиля, частота, період, модуль пружності, втрата стійкості, критична сила.

Purpose: to investigate the dynamics of cooperation of superficial layers of billet with an instrument at implementation of high-precision and power-hungry technological operations on treatment of metals pressure and tooling cutting, and also to execute the search of methods of intensification of the indicated working processes allowing to improve quality finish goods and lower power-hungriness of technological processes. 
Methods. For researches a model in that an instrument is conditionally presented as a hard stamp is accepted, and a detail is presented as a pliable flat stripe at the different terms of leaning of periblasts of the processed wares.

A hypothesis is in-process used that a nonlinear friction is the source of self-excited vibrations between an instrument and detail, that result in the origin of the overpriced efforts of cutting and receipt of treat surfaces, in a number of cases, high not enough quality.

Mathematical models are built for description of the deformed superficial layers of detail under act of the critical loading.

The methods of increase of efficiency of treatment of wares that is taken to application of imposition of vibrations with certain amplitudes and frequencies are recommended, thus, influence of these vibrations is taken to the conclusion and decision of equalizations consilient in due form with equalizations for self-excited vibrations.

Results. The problem of research of mechanics of co-operation of superficial layers of purveyance is in - process set and decided with an instrument at implementation of different technological operations taking into account description of friction of between contacting elements. For the set forth tasks on the processed surface there is a remaining wave that at further treatment is partly smoothed out by a tool piece. By authors, for the examined types of technological operations length of remaining deformation wave, amount of combs on this wave, and also value of critical force defiant this wave, is certain.

Originality. For description of process of dragging on wire-drawing options and process of tooling of wares on metal-cutting machine tools the same calculation model is applied and the same mathematical methods are used. For intensification of the examined technological processes it is recommended to use imposition of vibration with the optimal tuning on frequency and amplitude.

Practical implications. The indicated method of intensification of working processes at the optimal choice of parameters of the imposed vibrations can be successfully applied at dragging to the cold and hot rolling, stamping, treatment milling, planning, sharpening, crushing growing and boring drilling of mountain breeds and other technological processes.

Key words: dragging, rolling, rolling-up, stamping, purveyance, detail, milling, planning, description of friction, deformation wave, frequency, period, module of resiliency, loss of stability, critical force.

Рукопис надійшов 15.10.2019 